\title{
ASYMPTOTIC ANALYSIS, EXISTENCE AND SENSITIVITY RESULTS FOR A CLASS OF MULTIVALUED COMPLEMENTARITY PROBLEMS*
}

\author{
FABiÁn Flores-BazÁN ${ }^{1}$ AND RubÉn LÓPEZ ${ }^{2}$
}

\begin{abstract}
In this work we study the multivalued complementarity problem on the non-negative orthant. This is carried out by describing the asymptotic behavior of the sequence of approximate solutions to its multivalued variational inequality formulation. By introducing new classes of multifunctions we provide several existence (possibly allowing unbounded solution set), stability as well as sensitivity results which extend and generalize most of the existing ones in the literature. We also present some kind of robustness results regarding existence of solution with respect to certain perturbations. Topological properties of the solution-set multifunction are established and some notions of approximable multifunctions are also discussed. In addition, some estimates for the solution set and its asymptotic cone are derived, as well as the existence of solutions for perturbed problems is studied.
\end{abstract}

Mathematics Subject Classification. 90C33, 90C25, 47J20, 49J53.

Received October 7, 2004. Revised March 7, 2005.

\section{INTRODUCTION AND NOTATION}

A great variety of problems arising in most applications in Sciences and Engineering have the same mathematical formulation known as a multivalued complementarity problem which may be stated as follows: given a set-valued mapping, or simply a multifunction, $\Phi: \mathbb{R}_{+}^{n} \hookrightarrow \mathbb{R}^{n}$, and a vector $q \in \mathbb{R}^{n}$, it is requested to

$$
\text { find } \bar{x} \geq 0, \bar{y} \in \Phi(\bar{x}) \text { such that } \bar{y}+q \geq 0,\langle\bar{y}+q, \bar{x}\rangle=0 .
$$

This problem denoted by $\operatorname{MCP}(q, \Phi)$ generalizes substantially the so-called linear complementarity problem largely studied since 1958 (see [4], p. 218).

Problem (MCP) is known to be equivalent to the following multivalued variational inequality problem $\operatorname{MVIP}\left(\mathbb{R}_{+}^{n}, \Phi+q\right)$ :

$$
\text { find } \bar{x} \geq 0, \bar{y} \in \Phi(\bar{x}) \text { such that }\langle\bar{y}+q, x-\bar{x}\rangle \geq 0 \quad \forall x \geq 0 .
$$

In this paper we present a method which allows us to develop a general theory yielding new existence and sensitivity results and unifying the ones found in the literature. Our method is based on the asymptotic

\footnotetext{
Keywords and phrases. Multivalued complementarity problem, copositive mappings, asymptotic analysis, outer semicontinuity, graphical convergence.

* The research of the first author was supported by CONICYT-Chile through FONDECYT 104 -0610 and FONDAP-Matemáticas Aplicadas II. The second author was supported by Proyecto MECESUP UCO 9907 and FONDAP-Matemáticas Aplicadas II.

${ }^{1}$ Departamento de Ingeniería Matemática, Facultad de Ciencias Físicas y Matemáticas, Universidad de Concepción, Concepción, Chile; fflores@ing-mat.udec.cl

2 Facultad de Ingeniería, Universidad Católica de la Santísima Concepción, Concepción, Chile; rlopez@ucsc.cl
}

(C) EDP Sciences, SMAI 2006 
description of a sequence of approximate solutions to (MVIP). Thus, problems possibly allowing an unbounded solution set are also treated. Another advantage of our approach is that all requirements needed in this paper arise in a natural way. Several examples are discussed illustrating the wide applicability of our results. We follow the line of reasoning carried out in [7-9].

We end this section by stating some basic notations. Section 2 is devoted to list some definitions and preliminaries related to multifunctions. The asymptotic analysis of the approximate solutions to (MVIP) is performed in Section 3 (basic lemma) and the abstract Gowda-Pang existence theorem is reformulated therein. In Section 4, new classes of mappings are introduced and some of their properties are described. The main specializations of the abstract existence theorem are discussed in Section 5, where also some kind of robustness results are established. Section 6 is devoted to present some sensitivity and stability results by using the concept of graphical convergence, developed in [23]. Finally, precise bounds-estimations for the solution set are obtained in Section 7.

Throughout this paper, we will use the following notation: $x \geq 0$ (resp. $x>0$ ) whenever $x \in \mathbb{R}_{+}^{n}$ (resp. $\left.x \in \mathbb{R}_{++}^{n}=\operatorname{int} \mathbb{R}_{+}^{n}\right) ;|y|=\left(\left|y_{1}\right|, \ldots,\left|y_{n}\right|\right)$ and $\|y\|_{d}:=\langle d,|y|\rangle$ whenever $y \in \mathbb{R}^{n}$ and $d>0$ (in particular $\|y\|_{d}=\langle d, y\rangle$ for $\left.y \geq 0\right) ; \mathcal{S}(q, \Phi)$ stands for the solution set to $\operatorname{MCP}(q, \Phi) ; \operatorname{co} A$ is the convex hull of the set $A$; ri $A$ is the relative interior of $A$; $\operatorname{pos} A=\{t x: t \geq 0, x \in A\}$ is the positive hull of $A$; $\operatorname{pos}^{+} A=\{t x: t>0, x \in A\}$ is the strictly positive hull of $A ; A^{*}$ is the positive polar cone of $A ; A^{\#}$ is the strictly positive polar cone of $A$; given $x \in \mathbb{R}^{n}$, the index set $\operatorname{supp}\{x\}:=\left\{i \in I: x_{i} \neq 0\right\}$ is the support of $x$ where $I \doteq\{1, \ldots, n\}$; given $d>0$ we denote $\Delta_{d} \doteq\{x \geq 0:\langle d, x\rangle=1\}$ and if $J \subseteq I, \Delta_{J}=\Delta_{J}(d) \doteq \operatorname{co}\left\{\frac{1}{d_{i}} e^{i}: i \in J\right\}$ is an extreme face of $\Delta_{d}$, where $e^{i}$ is the $i$-th column of the identity matrix in $\mathbb{R}^{n \times n}$. In particular, $\Delta_{I}=\Delta_{d}$. The set

$$
\mathcal{D}(\Phi) \doteq\left\{q \in \mathbb{R}^{n}: q \in w-\Phi(x),(x, w) \in \mathbb{R}_{+}^{n} \times \mathbb{R}_{+}^{n},\langle w, x\rangle=0\right\}
$$

is the set of vectors for which $\operatorname{MCP}(q, \Phi)$ has solutions. More precisely,

$$
q \in \mathcal{D}(\Phi) \Longleftrightarrow \mathcal{S}(q, \Phi) \neq \emptyset \Longleftrightarrow \Phi \in \mathcal{D}^{-1}(q)
$$

Here $\mathcal{D}^{-1}$ denotes the inverse multifunction of $\mathcal{D}$ defined as usual. Moreover, the sets

$$
\mathcal{F}(q, \Phi) \doteq\{x \geq 0: y \in \Phi(x), y+q \geq 0\}, \mathcal{F}_{s}(q, \Phi) \doteq\{x \geq 0: y \in \Phi(x), y+q>0\}
$$

are the feasible and strict feasible sets of $\operatorname{MCP}(q, \Phi)$ respectively.

\section{Definitions AND PREliminaries}

Here and in the subsequent sections we will deal with multifunctions $\Phi$ defined in $\mathbb{R}_{+}^{n}$ which associates to any $x \in \mathbb{R}_{+}^{n}$ a nonempty set $\Phi(x)$ of $\mathbb{R}^{n}$, and denoted by $\Phi: \mathbb{R}_{+}^{n} \hookrightarrow \mathbb{R}^{n}$. The set gph $\Phi \doteq\left\{(x, y) \in \mathbb{R}_{+}^{n} \times \mathbb{R}^{n}: y \in \Phi(x)\right\}$ denotes the graph of $\Phi$.

A multifunction $\Phi: \mathbb{R}_{+}^{n} \hookrightarrow \mathbb{R}^{n}$ is said to be:

- compact (resp. convex) valued if for each $x \geq 0, \Phi(x)$ is compact (resp. convex);

- lower semicontinuous (lsc) if for any $x \geq 0, y \in \Phi(x)$ and any sequence $\left\{x^{k}\right\} \subseteq \mathbb{R}_{+}^{n}$ converging to $x$, there exists a sequence $\left\{y^{k}\right\}$ such that $y^{k} \in \Phi\left(x^{k}\right)$ and $y^{k} \rightarrow y$;

- upper semicontinuous (usc) if for each $x \geq 0$ and any open set $V \subseteq \mathbb{R}^{n}$ containing $\Phi(x)$, there is an open set $U \subseteq \mathbb{R}^{n}$ containing $x$ such that $\Phi\left(U \cap \mathbb{R}_{+}^{n}\right) \subseteq V$;

- a cusco if it is usc and compact convex valued;

- sequentially bounded if for any bounded sequence $\left\{x^{k}\right\} \subseteq \mathbb{R}_{+}^{n}$, it follows that any sequence $\left\{y^{k}\right\}$ with $y^{k} \in \Phi\left(x^{k}\right)$ for all $\mathrm{k}$, is bounded;

- superadditive if $\Phi(x)+\Phi(y) \subseteq \Phi(x+y)$ for all $x, y \geq 0$; 
- uniformly bounded if there exists a bounded set $C \subseteq \mathbb{R}^{n}$ such that $\Phi(x) \subseteq C$ for all $x \geq 0$;

- graph-convex (resp. graph-closed) if its graph is convex (resp. closed);

- $\mathbb{R}_{+}^{n}$-convex if $\lambda \Phi(x)+(1-\lambda) \Phi(y) \subseteq \Phi(\lambda x+(1-\lambda) y)+\mathbb{R}_{+}^{n}$ for all $x, y \geq 0, \lambda \in[0,1]$.

Before introducing our main classes of multifunctions, we need the following notation:

$$
\begin{gathered}
\mathcal{X} \doteq\left\{\Phi: \mathbb{R}_{+}^{n} \hookrightarrow \mathbb{R}^{n}: \Phi \text { is a cusco }\right\} \\
\mathcal{C} \doteq\left\{c: \mathbb{R}_{++} \rightarrow \mathbb{R}_{++}, c(0) \geq 0, \lim _{t \rightarrow+\infty} c(t)=+\infty\right\} .
\end{gathered}
$$

Definition 2.1. For $c \in \mathcal{C}, d>0$, the mapping $\Phi: \mathbb{R}_{+}^{n} \hookrightarrow \mathbb{R}^{n}$ such that $0 \in \Phi(0)$, is said to be

- $c$-homogeneous (on $\Delta_{d}$ ) if $\Phi(\lambda x)=c(\lambda) \Phi(x)$ for all $x \in \Delta_{d}$ and $\lambda>0$;

- $c$-subhomogeneous (on $\Delta_{d}$ ), if $\Phi(\lambda x) \subseteq c(\lambda) \Phi(x)$ for all $x \in \Delta_{d}$ and $\lambda>0$;

- zero-subhomogeneous (on $\Delta_{d}$ ), if $\Phi(\lambda x) \subseteq \Phi(x)$ for all $x \in \Delta_{d}$ and $\lambda>0$;

- $c$-Moré (on $\Delta_{d}$ ), if for all $\lambda \geq 1, x \in \Delta_{d}$, and $y \in \Phi(\lambda x)$ there exists $z \in \Phi(x)$ such that $\langle y, x\rangle \geq$ $c(\lambda)\langle z, x\rangle$.

We have to point out that our notion of (sub)homogeneity lies on the compact set $\Delta_{d}$ in place of the standard requirement lying on $\mathbb{R}_{+}^{n}$.

\section{Example 2.2.}

1. [13] An homogeneous multifunction $\Phi$ of degree $\gamma>0$, i.e. such that

$$
\Phi(\lambda x)=\lambda^{\gamma} \Phi(x) \text { for all } x \geq 0 \text { and } \lambda>0,
$$

is $\lambda^{\gamma}$-homogeneous on $\Delta_{d}$ for any $d>0$ provided $0 \in \Phi(0)$. For instance the mappings $\Phi_{1}(x)=M x$, where $M \in \mathbb{R}^{n \times n} ; \Phi_{2}(x)=\left(f_{1}(x), \ldots, f_{n}(x)\right)^{T}$, where $f_{i}(x)=\max \left\{\left\langle w_{i j}, x\right\rangle: j \in \Lambda_{i}\right\}$ with $w_{i j} \in \mathbb{R}^{n}$ and $\Lambda_{i}$ being a finite index set; $\Phi_{3}(x)=\{M y: A x+Q y \leq 0\}$, where $M \in \mathbb{R}^{n \times n}$ and $A, Q \in \mathbb{R}^{m \times n}$, are all homogeneous of degree 1. The mapping $\Phi_{4}(x)=\|x\| M x$ is homogeneous of degree 2 .

2. [25] A generalized homogeneous multifunction $\Phi$, i.e. such that for some $c \in \mathcal{C}$,

$$
\Phi(\lambda x)=c(\lambda) \Phi(x) \text { for all } x \geq 0 \text { and } \lambda>0,
$$

is $c$-homogeneous on $\Delta_{d}$ for any $d>0$ provided $0 \in \Phi(0)$.

3. The mappings $\Phi_{5}(x)=\left[x+x^{2}, 2 x+2 x^{2}\right]$ and $\Phi_{6}(x)=\left[e^{x}-1,2 e^{x}-2\right]$ are $c$-homogeneous on $\Delta_{1}$ with $c(\lambda)=\frac{\lambda+\lambda^{2}}{2}$ and $c(\lambda)=\frac{e^{\lambda}-1}{e-1}$ respectively, and if

$$
\Phi_{7}(x)=\left\{\begin{array}{ll}
{[0,1],} & \text { if } 0 \leq x \leq 1 ; \\
{[x-1, x],} & \text { if } x>1
\end{array} \text { and } \bar{c}(\lambda)= \begin{cases}1, & \text { if } 0 \leq \lambda \leq 1 \\
\lambda, & \text { if } \lambda>1\end{cases}\right.
$$

then $\Phi_{7}$ is $\bar{c}$-subhomogeneous on $\Delta_{1}$ but not $c$-homogeneous for any $c \in \mathcal{C}$.

4. $[18,19,25]$ Let $f: \mathbb{R}_{+}^{n} \rightarrow \mathbb{R}^{n}$ and $c \in \mathcal{C}$ such that

$$
\langle x, f(\lambda x)-f(0)\rangle \geq c(\lambda)\langle x, f(x)-f(0)\rangle \text { for all } x \geq 0 \text { and } \lambda \geq 1
$$

The mapping $\Phi(x)=f(x)-f(0)$ is $c$-Moré on $\Delta_{d}$ for any $d>0$. In connection with this result see (d) of Proposition 2.5 .

5. The mapping $\Phi_{8}(x)=[x, 2 x]$ is $\frac{\lambda}{2}$-Moré on $\Delta_{1}$.

6. The mapping $\Phi_{9}(x)=\left[0,1 /\|x\|_{d}\right]$ if $\|x\|_{d} \geq 1$ and $\Phi_{9}(x)=\left[0,\|x\|_{d}\right]$ if $\|x\|_{d} \leq 1$ is zero-subhomogeneous on $\Delta_{d}$ for $d>0$.

7. [13] An homogeneous multifunction $\Phi$ of degree 0 , i.e. such that

$$
\Phi(\lambda x)=\Phi(x) \text { for all } x \geq 0 \text { and } \lambda>0,
$$

is zero-subhomogeneous on $\Delta_{d}$ for any $d>0$ provided $0 \in \Phi(0)$. For instance, $\Phi_{10}(x)=\partial h(x)$ where $h(x)=$ $\sup _{y \in C}\langle x, y\rangle$ for $C \subseteq \mathbb{R}^{n}$ a nonempty compact convex set such that $0 \in C$. 
Proposition 2.3. Let $c \in \mathcal{C}$ and $\Phi: \mathbb{R}_{+}^{n} \hookrightarrow \mathbb{R}^{n}$ be a multifunction.

(a) If $\Phi$ is usc with compact values, then it is sequentially bounded and graph-closed.

(b) If $\Phi$ is a zero-subhomogeneous cusco, then it is uniformly bounded.

(c) If $\Phi$ is either graph-convex or c-homogeneous with $c\left(\mathbb{R}_{+}\right)=\mathbb{R}_{+}, \Phi(0)=\{0\}$, and superadditive, then $\Phi\left(\mathbb{R}_{+}^{n}\right)$ is convex.

Proof.

(a) It follows from Propositions 1.1.3 and 1.1.2 in [1].

(b) For $0 \neq x \geq 0$, we have $\Phi(x)=\Phi\left(\|x\|_{d} \frac{x}{\|x\|_{d}}\right) \subseteq \Phi\left(\frac{x}{\|x\|_{d}}\right) \subseteq \Phi\left(\Delta_{d}\right)$, which implies the desired result since $\Phi\left(\Delta_{d}\right)$ is compact [1], Proposition 1.1.3.

(c) It is obvious.

Notice that within cuscos mappings, uniformly bounded does not imply zero-subhomogeneity as the function $\Phi(x)=1 /(1+x)$ shows.

The (nonlinear) multivalued version of the classes of mappings introduced in the study of linear complementarity problems (see $[4,9]$ for example) arise in a natural way in the present setting. We now recall some of them. Let $d>0$ and $\Phi: \mathbb{R}_{+}^{n} \hookrightarrow \mathbb{R}^{n}$ be a multifunction. We say that $\Phi$ is:

- copositive if $\langle y, x\rangle \geq 0 \quad \forall(x, y) \in \operatorname{gph} \Phi$;

- strictly copositive if $\langle y, x\rangle>0 \quad \forall(x, y) \in \operatorname{gph} \Phi$ with $x \neq 0$;

- strongly copositive if $\exists \alpha>0$ such that $\langle y, x\rangle \geq \alpha\|x\|^{2} \quad \forall(x, y) \in \operatorname{gph} \Phi$;

- semimonotone if $\mathcal{S}(p, \Phi)=\{0\} \quad \forall p>0$;

- a $\mathbf{R}(d)$-mapping, or $\Phi \in \mathbf{R}(d)$, if $\mathcal{S}(\tau d, \Phi)=\{0\} \quad \forall \tau \geq 0$;

- a $\mathbf{G}(d)$-mapping, or $\Phi \in \mathbf{G}(d)$, if $\mathcal{S}(\tau d, \Phi)=\{0\} \quad \forall \tau>0$;

- monotone if for any $\left(x^{1}, y^{1}\right),\left(x^{2}, y^{2}\right) \in \operatorname{gph} \Phi,\left\langle y^{1}-y^{2}, x^{1}-x^{2}\right\rangle \geq 0$;

- q-pseudomonotone if for any $\left(x^{1}, y^{1}\right),\left(x^{2}, y^{2}\right) \in \operatorname{gph} \Phi$,

$$
\left\langle y^{1}+q, x^{2}-x^{1}\right\rangle \geq 0 \Longrightarrow\left\langle y^{2}+q, x^{2}-x^{1}\right\rangle \geq 0 .
$$

The following definition generalizes that for linear mappings used in [14].

Definition 2.4. For $d>0$ and $\Phi \in \mathcal{X}$. The $d$-numerical range of $\Phi$ is by definition, the set

$$
\omega(\Phi) \doteq\left\{\langle y, x\rangle: x \in \Delta_{d}, y \in \Phi(x)\right\} .
$$

We set $M_{\Phi} \doteq \sup \omega(\Phi)$ and $m_{\Phi} \doteq \inf \omega(\Phi)$.

Proposition 2.5. Let $d>0, c \in \mathcal{C}$, and $\Phi \in \mathcal{X}$.

(a) If $\Phi$ is c-subhomogeneous and $m_{\Phi}>0$ (in particular if $\Phi$ is strictly copositive), then $\Phi$ is $\bar{c}$-Moré for $\bar{c}(\lambda)=\frac{m_{\Phi}}{M_{\Phi}} c(\lambda)$.

(b) If $\Phi$ is copositive c-subhomogeneous and the following implication holds: $(v \geq 0, w \in \Phi(v),\langle w, v\rangle=0 \Longrightarrow v=0)$, then $\Phi$ is $\bar{c}$-Moré.

(c) If $\Phi$ is strongly copositive and $0 \in \Phi(0)$, then it is $\tilde{c}$-Moré for $\tilde{c}(\lambda)=\frac{\alpha \lambda}{M_{\Phi}\|d\|^{2}}$.

(d) If $\Phi$ is $\mathbb{R}_{+}^{n}$-convex and $0 \in \Phi(0)$, then it is $\hat{c}$-Moré for $\hat{c}(\lambda)=\lambda$.

Proof.

(a) Let $\lambda \geq 1, x \in \Delta_{d}$, and $y \in \Phi(\lambda x)$ be given, by hypothesis $\frac{1}{c(\lambda)} y \in \Phi(x)$ and for any $z \in \Phi(x)$ we get $\left\langle\frac{1}{c(\lambda)} y, x\right\rangle \geq m_{\Phi} \geq \frac{m_{\Phi}}{M_{\Phi}}\langle z, x\rangle$. Thus, setting $\bar{c}(\lambda)=\frac{m_{\Phi}}{M_{\Phi}} c(\lambda)$ we obtain the desired result.

(b) Since $\Phi$ is copositive, $m_{\Phi} \geq 0$. Suppose that $m_{\Phi}=0$, then there exist $x \in \Delta_{d}$ and $y \in \Phi(x)$ such that $m_{\Phi}=\langle y, x\rangle=0$, contradicting the hypothesis. The result follows from (a).

(c) Let $\lambda \geq 1$ and $x \in \Delta_{d}$, then $\|x\| \geq \frac{1}{\|d\|}$ and if $y \in \Phi(\lambda x)$, then $\langle y, \lambda x\rangle \geq \alpha\|\lambda x\|^{2}$ for some $\alpha>0$, and thus $\langle y, x\rangle \geq \frac{\alpha \lambda}{\|d\|^{2}}$. Clearly if $z \in \Phi(x)$ then $\frac{1}{M_{\Phi}}\langle z, x\rangle \leq 1$. Therefore $\langle y, x\rangle \geq \frac{\alpha \lambda}{\|d\|^{2}} \geq \tilde{c}(\lambda)\langle z, x\rangle$. 
(d) Let $\lambda \geq 1$ and $x \in \Delta_{d}$, by definition $\frac{1}{\lambda} \Phi(\lambda x)+\left(1-\frac{1}{\lambda}\right) \Phi(0) \subseteq \Phi(x)+\mathbb{R}_{+}^{n}$, since $0 \in \Phi(0)$ we conclude that $\frac{1}{\lambda} \Phi(\lambda x) \subseteq \Phi(x)+\mathbb{R}_{+}^{n}$, thus, if $y \in \Phi(\lambda x)$ then there exists $z \in \Phi(x)$ such that $\frac{1}{\lambda} y \geq z$. Thus, $\langle y, x\rangle \geq$ $\hat{c}(\lambda)\langle z, x\rangle$.

\section{Asymptotic Analysis And the ABStract eXistence theorem}

We approximate problem (MVIP), which is the variational inequality formulation to (MCP), by the following sequence of problems

$$
\text { find } x^{k} \in D_{k}, y^{k} \in \Phi\left(x^{k}\right):\left\langle y^{k}+q, x-x^{k}\right\rangle \geq 0 \forall x \in D_{k}
$$

where $d>0,\left\{\sigma_{k}\right\}$ is an increasing sequence of positive numbers converging to $+\infty$, and

$$
D_{k}=\left\{x \in \mathbb{R}_{+}^{n}:\langle d, x\rangle \leq \sigma_{k}\right\} .
$$

If $\Phi$ is usc with nonempty compact convex values, the existence of $\left(x^{k}, y^{k}\right) \in \operatorname{gph} \Phi$ satisfying $\left(\mathrm{MVIP}_{\mathrm{k}}\right)$ is guaranteed by the multivalued version of the classical Hartman-Stampacchia existence theorem (see [13] for instance).

It is clear that $\left(x^{k}, y^{k}\right)$ solves $\left(\mathrm{MVIP}_{\mathrm{k}}\right)$ if and only if $x^{k}$ is an optimal solution of the linear program

$$
\inf _{x}\left[\left\langle y^{k}+q, x\right\rangle: x \geq 0,\langle d, x\rangle \leq \sigma_{k}\right] .
$$

Applying the usual optimality conditions we conclude that $\left(x^{k}, y^{k}\right)$ solves $\left(\mathrm{MVIP}_{\mathrm{k}}\right)$ if and only if there exists $\theta_{k} \in \mathbb{R}$ such that $\left(x^{k}, y^{k}, \theta_{k}\right)$ solves the so-called augmented multivalued complementarity problem

$$
\begin{gathered}
\text { find } x^{k} \geq 0, \theta_{k} \geq 0, y^{k} \in \Phi\left(x^{k}\right) \text { such that } \\
y^{k}+q+\theta_{k} d \geq 0, \quad\left\langle d, x^{k}\right\rangle \leq \sigma_{k}, \\
\left\langle y^{k}+q+\theta_{k} d, x^{k}\right\rangle=0, \quad \theta_{k}\left(\sigma_{k}-\left\langle d, x^{k}\right\rangle\right)=0 .
\end{gathered}
$$

In particular,

$$
x^{k} \in \mathcal{S}\left(q+\theta_{k} d, \Phi\right) \text { and } x^{k} \in \mathcal{S}\left(q, \Phi+\theta_{k} d\right) .
$$

Clearly, we observe that

$$
\left\langle d, x^{k}\right\rangle<\sigma_{k} \Longrightarrow \theta_{k}=0 \Longrightarrow x^{k} \in \mathcal{S}(q, \Phi) .
$$

This line of reasoning was also applied in [22].

We introduce the following definition: a subset $M$ of a metric space $X$ is said to be closed at $x$, if whenever a sequence $\left\{x^{k}\right\} \subseteq M$ converges to $x$, one has $x \in M$. Obviously, if $M$ is closed then $M$ is closed at every point $x \in M$.

The next theorem was established in [13].

Theorem 3.1. Let $d>0,\left\{\sigma_{k}\right\}$ be an increasing sequence of positive numbers converging to $+\infty, \Phi \in \mathcal{X}$, and $\left\{\left(x^{k}, y^{k}, \theta_{k}\right)\right\}$ be a sequence of solutions to problem $\left(\mathrm{MCP}_{\mathrm{k}}\right)$. Assume $\liminf _{k \rightarrow+\infty} \theta_{k}=0$. Then, the following assertions are equivalent:

(a) $\mathcal{S}(q, \Phi)$ is nonempty;

(b) $\mathcal{D}(\Phi)$ is closed at $q$.

Proof.

(a) $\Rightarrow(\mathrm{b})$ : it is obvious.

(b) $\Rightarrow\left(\right.$ a): without loss of generality we may assume that $\theta_{k} \rightarrow 0$. By (3.1), we conclude that $\mathcal{S}\left(q+\theta_{k} d, \Phi\right)$ is nonempty, thus $q+\theta_{k} d \in \mathcal{D}(\Phi)$. By hypothesis $q \in \mathcal{D}(\Phi)$, thus $\mathcal{S}(q, \Phi)$ is nonempty. 
An important class of multifunctions $\Phi$ for which $\mathcal{D}(\Phi)$ is closed at every $q$ is that of polyhedral ones (see for instance [13]). However we look for new classes of multifunctions such that $\mathcal{D}(\Phi)$ is closed at some particular $q$. These classes are introduced in Section 4.

Remark 3.2. As pointed above, if $\theta_{k}=0$ for some $k$, then $\mathcal{S}(q, \Phi)$ is nonempty. Theorem 3.1 yields existence of solutions when $\theta_{k}>0$ for all $k$. Indeed, let $\Phi\left(x_{1}, x_{2}\right)=\left[-x_{1}, x_{1}\right] \times\left[-x_{2}, x_{2}\right], d=(1,1)^{\mathrm{T}}, \sigma_{k}=k$, and $q=(0,-1)^{\mathrm{T}}$. We have that $\left\{\left(x^{k}, y^{k}, \theta_{k}\right)\right\}$ solves $\left(\mathrm{MCP}_{\mathrm{k}}\right)$ for $x^{k}=(0, k)^{\mathrm{T}}, y^{k}=\left(0,1-\frac{1}{k}\right)^{\mathrm{T}}$, and $\theta_{k}=\frac{1}{k}>0$. Since $\liminf _{k \rightarrow+\infty} \theta_{k}=0$ and $\mathcal{D}(\Phi)$ is closed being $\Phi$ polyhedral (see [13], Prop. 3), the above theorem asserts that $\mathcal{S}(q, \Phi)$ is nonempty. Indeed, $\mathcal{S}(q, \Phi)=\left\{\left(x_{1}, x_{2}\right)^{\mathrm{T}}: x_{1} \geq 0, x_{2} \geq 1\right\}$.

In our opinion, Theorem 3.1 was established only by taking $x^{k} \in \mathcal{S}\left(q+\theta_{k} d, \Phi\right)$ into account in (3.1); in this respect the closedness of $\mathcal{D}(\Phi)$ at $q$ plays a certain role since $q+\theta^{k} d \in \mathcal{D}(\Phi)$ and, $\mathcal{S}(q, \Phi) \neq \emptyset \Longleftrightarrow q \in \mathcal{D}(\Phi)$. However, if instead we look at $x^{k} \in \mathcal{S}\left(q, \Phi+\theta_{k} d\right)$ in $(3.1)$, we have to analize the closedness of $\mathcal{D}^{-1}(q)$ relative to some particular class of approximating mappings. Thus, we first need a good notion of convergence for multifunctions, and secondly, to find the particular approximating mappings. Just to give an idea to be developed presently, we observe that the $c$-subhomogeneity of $\Phi$ does not imply the $c$-subhomegeneity of $\Phi+\theta_{k} d$; however, the multifunction $\Psi^{k}$ given by $\Psi^{k}(x)=\theta_{k} d$ is copositive and uniformly bounded whenever $\theta_{k} \rightarrow 0$. On the other hand, in order to discuss sensitivity and stability results we also substitute $\Phi$ by an approximating mapping $\Phi^{k}$. These considerations give rise to the notion of approximable mappings to be discussed in Section 6 .

The next (basic) lemma describes the asymptotic behavior of the corresponding solutions to the problems associated to the approximating mappings (see $\left(\mathrm{PMVIP}_{\mathrm{k}}\right)$ below). Existence of solutions will require further assumptions on the original and/or the approximating mappings. The latter is also analyzed in Section 6.

We start by introducing the notion of convergence for multifunctions. To that end, we need the following concepts (for more details see [23], Ch. 4). For a nonempty set $C \subseteq \mathbb{R}^{n}, d_{C}(x) \doteq d(C, x)$ stands for the distance from $x$ to $C$. Let $A, B \subseteq \mathbb{R}^{n}$ be two nonempty sets, the integrated set distance between them is defined by

$$
\mathrm{d} I(A, B) \doteq \int_{0}^{\infty} \mathrm{d} I_{\rho}(A, B) \mathrm{e}^{-\rho} \mathrm{d} \rho
$$

where for $\rho \geq 0$,

$$
\mathrm{dI}_{\rho}(A, B) \doteq \max _{\|x\| \leq \rho}\left|d_{A}(x)-d_{B}(x)\right| .
$$

The expression $\mathrm{dI}$ gives a metric on cl - sets $\neq \emptyset\left(\mathbb{R}^{n}\right)$-the space of all nonempty closed subsets of $\mathbb{R}^{n}$, and characterizes the ordinary set convergence in the sense of Painlevé-Kuratowski, i.e. $C^{k} \rightarrow C \Longleftrightarrow \mathrm{dI}\left(C^{k}, C\right) \rightarrow 0$.

On $\mathcal{X}$ we consider the metric (we shall denote also by dI)

$$
\mathrm{d}\left(\Phi^{1}, \Phi^{2}\right) \doteq \mathrm{dI}\left(\operatorname{gph} \Phi^{1}, \operatorname{gph} \Phi^{2}\right),
$$

which characterizes the graphical convergence $\stackrel{g}{\rightarrow} "$, i.e.

$$
\Phi^{k} \stackrel{g}{\rightarrow} \Phi \Longleftrightarrow \mathrm{dI}\left(\Phi^{k}, \Phi\right) \rightarrow 0
$$

and will be crucial in our analysis.

The following lemma, which is important on its own, will be repeatedly used in the subsequent sections. This basic lemma will be extremely useful for deriving existence, sensitivity as well as stability results.

Lemma 3.3 (basic lemma). Let $d>0,\left\{\sigma_{k}\right\}$ be an increasing sequence of positive numbers converging to $+\infty$; $q, q^{k} \in \mathbb{R}^{n} ; \Phi, \Psi, \Phi^{k}, \Psi^{k} \in \mathcal{X}$ be such that $\Phi^{k} \stackrel{g}{\rightarrow} \Phi, \Psi^{k} \stackrel{g}{\rightarrow} \Psi, q^{k} \rightarrow q$ and $\left\{\left(x^{k}, y^{k}, r^{k}\right)\right\}$ be a sequence of solutions to

$$
\text { find } x^{k} \in D_{k}: y^{k} \in \Phi^{k}\left(x^{k}\right), r^{k} \in \Psi^{k}\left(x^{k}\right),\left\langle y^{k}+r^{k}+q^{k}, x-x^{k}\right\rangle \geq 0 \forall x \in D_{k} .
$$


such that $\left\langle d, x^{k}\right\rangle=\sigma_{k}$ and $\frac{x^{k}}{\sigma_{k}} \rightarrow v$ as $k \rightarrow+\infty$. Then, there exist subsequences $\left\{\sigma_{k_{m}}\right\}$ and $\left\{\left(x^{k_{m}}, y^{k_{m}}, r^{k_{m}}\right)\right\}$, numbers $k_{0}, m_{0} \in \mathbb{N}$, and an index set $\emptyset \neq J_{v} \subseteq I$ such that

(a) for all $k \geq k_{0}, x^{k}-\frac{\sigma_{k}}{2} v \geq 0$ and $0<\left\|x^{k}-\frac{\sigma_{k}}{2} v\right\|_{d}<\sigma_{k}$;

(b) for all $m \geq m_{0}, \frac{1}{\sigma_{k_{m}}} x^{k_{m}} \in \operatorname{ri}\left(\Delta_{J_{v}}\right)$, thus $\operatorname{supp}\left\{x^{k_{m}}\right\}=J_{v}$ (hence $\left.\operatorname{supp}\{v\} \subseteq J_{v}\right)$;

(c) for all $m \geq m_{0}, z \in \Delta_{J_{v}}:\left\langle y^{k_{m}}+r^{k_{m}}+q^{k_{m}}, \sigma_{k_{m}} z-x^{k_{m}}\right\rangle=0$.

Moreover,

(d) if each $\Phi^{k}$ is c-subhomogeneous and each $\Psi^{k}$ is uniformly bounded respect to the same set (resp. zerosubhomogeneous), then the subsequences $\left\{y^{k_{m}}\right\},\left\{r^{k_{m}}\right\},\left\{\sigma_{k_{m}}\right\}$ may be chosen in such a way that there are vectors $w$ and $r$ such that $\frac{1}{c\left(\sigma_{k_{m}}\right)} y^{k_{m}} \rightarrow w \in \Phi(v), r^{k_{m}} \rightarrow r($ resp. and $r \in \Psi(v)),\langle w, v\rangle \leq 0$, $\langle w, y\rangle \geq\langle d, y\rangle\langle w, v\rangle$ for all $y \geq 0$, and $\langle w, z\rangle=\langle w, v\rangle$, for all $z \in \Delta_{J_{v}}$

(e) if each $\Phi^{k}$ is c-Moré and each $\Psi^{k}$ is uniformly bounded respect to the same set, then there exist vectors $w, r$ and sequences $\left\{w^{k_{m}}\right\}$ and $\left\{r^{k_{m}}\right\}$ such that $w^{k_{m}} \in \Phi^{k_{m}}\left(\frac{x^{k_{m}}}{\sigma_{k_{m}}}\right), w^{k_{m}} \rightarrow w \in \Phi(v), r^{k_{m}} \rightarrow r$, and $\langle w, v\rangle \leq 0$

(f) if each $\Phi^{k}$ is $q^{k}$-pseudomonotone and each $\Psi^{k}=0$, then $v \in \mathbb{R}_{+}^{n} \cap\left[-\Phi\left(\mathbb{R}_{+}^{n}\right)-q\right]^{*}$. Hence, $0 \leq v \in$ $-\left[\Phi\left(\mathbb{R}_{+}^{n}\right)\right]^{*}$ and $\langle q, v\rangle \leq 0$ provided $\Phi$ is c-homogeneous and $\Phi(0)=\{0\}$ as well;

(g) if each $\Phi^{k}$ is monotone and each $\Psi^{k}$ is copositive zero-subhomogeneous, then

$$
v \in \mathbb{R}_{+}^{n} \cap\left[-\Phi\left(\mathbb{R}_{+}^{n}\right)-q\right]^{*} .
$$

Proof. By Theorems 5.19 and 5.51(b) from [23], $\Phi^{k}+\Psi^{k} \in \mathcal{X}$, and problem (PMVIP ${ }_{\mathrm{k}}$ ) has solutions by the multivalued version of Hartman-Stampacchia Theorem.

(a) As $\frac{1}{\sigma_{k}} x^{k} \rightarrow v$, for $\varepsilon=\min \left\{\frac{v_{i}}{2}: v_{i}>0\right\}>0$ there exists $k_{0}$ such that for all $k \geq k_{0}, \sum_{i=1}^{n}\left|\frac{x_{i}^{k}}{\sigma_{k}}-v_{i}\right|<\varepsilon$. This implies $\frac{v_{i}}{2}<\frac{x_{i}^{k}}{\sigma_{k}}$ for $i \in \operatorname{supp}\{v\}$. Thus $0 \neq x^{k}-\frac{\sigma_{k}}{2} v \geq 0$, and then (a) holds.

(b) Clearly $\Delta_{d}=\Delta_{I}=\operatorname{co}\left\{\frac{1}{d_{i}} e_{i}: i \in I\right\}$ may be written as the disjoint union of the relative interior of its extreme faces. More precisely, if we denote its extreme faces by $\Delta_{J_{1}}, \Delta_{J_{2}}, \ldots, \Delta_{J_{2^{n}-1}}$, then

$$
\Delta_{d}=\bigcup_{i=1}^{2^{n}-1} \operatorname{ri}\left(\Delta_{J_{i}}\right) .
$$

As $\frac{1}{\sigma_{k}} x^{k} \in \Delta_{d}, k \in \mathbb{N}$, there exist an $i_{0} \in\left\{1,2, \ldots, 2^{n}-1\right\}, m_{0}$, and a subsequence $\left\{x^{k_{m}}\right\}$ such that $\frac{1}{\sigma_{k_{m}}} x^{k_{m}} \in \operatorname{ri}\left(\Delta_{J_{i_{0}}}\right)$ for all $m \geq m_{0}$. By setting $J_{v} \doteq J_{i_{0}}$, one obtains $\operatorname{supp}\left\{x^{k_{m}}\right\}=J_{v}$ and $\operatorname{supp}\{v\} \subseteq J_{v}$.

(c) We analyze two cases, whether $J_{v}$ is a singleton or not. In the first case, we have $\frac{1}{\sigma_{k_{m}}} x^{k_{m}}=v$ for all $m \geq m_{0}$ because of $\operatorname{ri}\left(\Delta_{J_{v}}\right)=\Delta_{J_{v}}$, and therefore (c) obviously holds. In the second case, for all $z \in \Delta_{J_{v}}$ and all $m \geq m_{0}$, by virtue of (b) there exists $\varepsilon_{z}>0$ such that for all $t,|t|<\varepsilon_{z}$, one obtains

$$
\frac{1}{\sigma_{k_{m}}} x^{k_{m}}+t\left(z-\frac{1}{\sigma_{k_{m}}} x^{k_{m}}\right) \in \Delta_{J_{v}} .
$$

Because of the choice of $x^{k_{m}}$, we have

$$
\left\langle y^{k_{m}}+r^{k_{m}}+q^{k_{m}}, \sigma_{k_{m}}\left(\frac{x^{k_{m}}}{\sigma_{k_{m}}}+t\left(z-\frac{x^{k_{m}}}{\sigma_{k_{m}}}\right)\right)-x^{k_{m}}\right\rangle \geq 0, \quad \forall|t|<\varepsilon_{z} .
$$

Then

Hence

$$
\left\langle y^{k_{m}}+r^{k_{m}}+q^{k_{m}}, t\left(\sigma_{k_{m}} z-x^{k_{m}}\right)\right\rangle \geq 0, \quad \forall|t|<\varepsilon_{z} .
$$

$$
\left\langle y^{k_{m}}+r^{k_{m}}+q^{k_{m}}, \sigma_{k_{m}} z-x^{k_{m}}\right\rangle=0, \quad \forall z \in \Delta_{J_{v}} .
$$


(d) By assumption $\frac{y^{k}}{c\left(\sigma_{k}\right)} \in \Phi^{k}\left(\frac{x^{k}}{\sigma_{k}}\right)$. Since $\left\{\frac{x^{k}}{\sigma_{k}}\right\}$ is bounded, by the uniformity in graphical convergence $\Phi^{k} \stackrel{g}{\rightarrow} \Phi[23]$, Theorem 5.34, we may also assume that $\frac{y^{k}}{c\left(\sigma_{k}\right)} \rightarrow w$ up to subsequences. From (a) of Theorem 5.37 in [23], it follows in particular that $w \in \Phi(v)$.

Moreover, from $r^{k} \in \Psi^{k}\left(x^{k}\right)$, if $\Psi^{k}$ is zero-subhomogeneous, $r^{k} \in \Psi^{k}\left(\frac{x^{k}}{\sigma_{k}}\right)$, and reasoning as above (up to subsequences) $r^{k} \rightarrow r$, and $r \in \Psi(v)$. On the other hand, if $\Psi^{k}$ is uniformly bounded respect to the same set, the sequence $\left\{r^{k}\right\}$ is bounded and up to a subsequence $r^{k} \rightarrow r$.

For both cases, on dividing the inequality in $\left(\mathrm{PMVIP}_{\mathrm{k}}\right)$ by $c\left(\sigma_{k}\right) \sigma_{k}$ and letting $k \rightarrow+\infty$ for $x=0$ and $x=\sigma_{k} \frac{y}{\|y\|_{d}}$ with $0 \neq y \geq 0$ respectively, we obtain $\langle w, v\rangle \leq 0$ and $\langle w, y\rangle \geq\langle d, y\rangle\langle w, v\rangle$ for all $y \geq 0$. Dividing (c) by $c\left(\sigma_{k_{m}}\right) \sigma_{k_{m}}$ and letting $m \rightarrow+\infty$ we obtain the last part of (d).

(e) By assumption, $\left\langle y^{k}, \frac{x^{k}}{\sigma_{k}}\right\rangle \geq c\left(\sigma_{k}\right)\left\langle w^{k}, \frac{x^{k}}{\sigma_{k}}\right\rangle$ for some $w^{k} \in \Phi^{k}\left(\frac{x^{k}}{\sigma_{k}}\right)$. As in (d), we may suppose up to subsequences that $w^{k} \rightarrow w$ and $w \in \Phi(v)$. On dividing $\left(\mathrm{PMVIP}_{\mathrm{k}}\right)$ (for $\left.x=0\right)$ by $c\left(\sigma_{k}\right)$, we get

$$
-\left\langle\frac{r^{k}+q^{k}}{c\left(\sigma_{k}\right)}, \frac{x^{k}}{\sigma_{k}}\right\rangle \geq\left\langle\frac{y^{k}}{c\left(\sigma_{k}\right)}, \frac{x^{k}}{\sigma_{k}}\right\rangle \geq\left\langle w^{k}, \frac{x^{k}}{\sigma_{k}}\right\rangle .
$$

Taking the limit we obtain $\langle w, v\rangle \leq 0$.

(f) Let us fix $x \geq 0$ and $y \in \Phi(x)$. Since $\Phi^{k}, \Phi$ are closed-valued and $\Phi^{k} \stackrel{g}{\rightarrow} \Phi$, we invoke Theorem 5.37 in [23] to obtain $x$ as the limit of a sequence $\left\{a^{j}\right\} \subseteq \mathbb{R}_{+}^{n}$, corresponding to some choice of $\left\{b^{j}\right\}$ satisfying $b^{j} \in \Phi^{j}\left(a^{j}\right)$ and $b^{j} \rightarrow y$ as $j \rightarrow+\infty$. Obviously there is $j_{0}$ such that $a^{j} \in D_{j_{0}}$ for all $j$. In particular, for $j \geq j_{0}$ we have that $a^{j} \in D_{j_{0}} \subseteq D_{j}$. By $q^{j}$-pseudomonotonicity of $\Phi^{j},\left(\mathrm{PMVIP}_{\mathrm{j}}\right)$ implies $\left\langle b^{j}+q^{j}, a^{j}-x^{j}\right\rangle \geq 0$ for all $j$ sufficiently large, dividing by $\sigma_{j}$ and taking the limit we conclude that $\langle y+q, v\rangle \leq 0$. Thus $v \in\left[-\Phi\left(\mathbb{R}_{+}^{n}\right)-q\right]^{*}$. The remaining part is obvious.

(g) From $r^{k} \in \Psi^{k}\left(x^{k}\right)$, if $\Psi^{k}$ is zero-subhomogeneous $r^{k} \in \Psi^{k}\left(\frac{x^{k}}{\sigma_{k}}\right)$ and as in (d), up to subsequences $r^{k} \rightarrow r$ and $r \in \Psi(v)$. By hypothesis $\langle r, v\rangle \geq 0$.

Let us fix $x \geq 0$ and $y \in \Phi(x)$, as in (f) we obtain $x$ as the limit of a sequence $\left\{a^{j}\right\} \subseteq \mathbb{R}_{+}^{n}$, corresponding to some choice of $\left\{b^{j}\right\}$ satisfying $b^{j} \in \Phi^{j}\left(a^{j}\right)$ and $b^{j} \rightarrow y$ as $j \rightarrow+\infty$, and $a^{j} \in D_{j_{0}} \subseteq D_{j}$ for $j \geq j_{0}$. By $\left(r^{j}+q^{j}\right)$-pseudomonotonicity of $\Phi^{j},\left(\mathrm{PMVIP}_{\mathrm{j}}\right)$ implies $\left\langle b^{j}+r^{j}+q^{j}, a^{j}-x^{j}\right\rangle \geq 0$ for all $j$ sufficiently large; dividing by $\sigma_{j}$ and taking the limit we conclude that $0 \geq\langle y+r+q, v\rangle \geq\langle y+q, v\rangle$. Thus $v \in\left[-\Phi\left(\mathbb{R}_{+}^{n}\right)-q\right]^{*}$.

Remark 3.4. Clearly $\langle d, v\rangle=1$. Moreover, when $\Phi^{k}$ are $c$-subhomogeneous, by choosing $y=e^{i}, i \in I$ in $(\mathrm{d})$ and setting $\tau=-\langle w, v\rangle \geq 0$, we obtain $w+\tau d \geq 0$ and $\langle w+\tau d, v\rangle=0$. Thus $0 \neq v \in \mathcal{S}(\tau d, \Phi)$.

We now exhibit an instance where our basic lemma is applicable. Let us consider $\Phi^{k}(x)=M^{k} x, \Psi^{k}(x)=$ $\partial \sigma_{C^{k}}(x)$, where $M^{k} \in \mathbb{R}^{n \times n}$ converges to $M \in \mathbb{R}^{n \times n}$, and $\partial \sigma_{C^{k}}$ is the (Fenchel) subdifferential of the support function of the nonempty compact convex set $C^{k}$, which converges (in the sense of Painlevé-Kuratowski) to the nonempty compact convex $C$. It is known that $M^{k} \stackrel{g}{\rightarrow} M$ (see [23], p. 353), and by Corollaries 11.5 and 8.24, and Theorem 12.35 of [23], one obtains, $C^{k} \rightarrow C \Longleftrightarrow \partial \sigma_{C^{k}} \stackrel{g}{\rightarrow} \partial \sigma_{C}$. Moreover, as mentioned in Example 2.2, each $\partial \sigma_{C^{k}}$ and $\partial \sigma_{C}$ is zero-subhomogeneous. In addition, if $0 \in C^{k} \cap C$ (this is not a stringent assumption), then $\partial \sigma_{C^{k}}$ and $\partial \sigma_{C}$ are copositive.

Let $\left\{\left(x^{k}, y^{k}\right)\right\}$ be a sequence of solutions to $\left(\mathrm{MVIP}_{\mathrm{k}}\right)$, if $\left\langle d, x^{k}\right\rangle<\sigma_{k}$ for some $k$, then $x^{k} \in \mathcal{S}(q, \Phi)$. Thus, we are interested in the case when $\left\langle d, x^{k}\right\rangle=\sigma_{k}$ for all $k$. Therefore, the following set of sequences will play an essential role in our analysis.

Definition 3.5. Let $d>0$ and $\left\{\sigma_{k}\right\}$ be an increasing sequence of positive numbers converging to $+\infty$. Let $\mathcal{W}$ be the set of sequences $\left\{\left(x^{k}, y^{k}\right)\right\}$ in $\mathbb{R}_{+}^{n} \times \mathbb{R}^{n}$, satisfying for each $k$,

$$
\begin{gathered}
\left(x^{k}, y^{k}\right) \text { solves problem }\left(\operatorname{MVIP}_{\mathrm{k}}\right) \\
\left\langle d, x^{k}\right\rangle=\sigma_{k} .
\end{gathered}
$$


We point out that the requirement (3.3) is verified if $\mathcal{S}(q, \Phi)$ is either empty or unbounded. Indeed, if $\mathcal{S}(q, \Phi)=\emptyset$ then $\left\langle d, x^{k}\right\rangle=\sigma_{k}$ for all $k$ by the above reasoning. If $\mathcal{S}(q, \Phi)$ is nonempty and unbounded, then for all $k$, there exists $x^{k} \in \mathcal{S}(q, \Phi)$ such that $\left\langle d, x^{k}\right\rangle \geq k$, and then we put $\sigma_{k}=\left\langle d, x^{k}\right\rangle$.

Under requirement (3.3), there exists a vector $v$ such that, up to subsequences, $\frac{x^{k}}{\sigma_{k}} \rightarrow v$, and if $\left\{\theta_{k}\right\}$ is such that $\left(x^{k}, y^{k}, \theta_{k}\right)$ solves $\left(\mathrm{MCP}_{\mathrm{k}}\right)$ for each $k$, by (c) of the basic lemma (for $\Phi^{k}=\Phi, \Psi^{k}=0$ and $q^{k}=q$ for all $k$ ) we get.

$$
\theta_{k_{m}}=-\left\langle y^{k_{m}}+q, \frac{x^{k_{m}}}{\sigma_{k_{m}}}\right\rangle=-\left\langle y^{k_{m}}+q, v\right\rangle
$$

\section{New Classes of Mappings ANd estimates for $[S(q, \Phi)]^{\infty}$}

We introduce the following classes of mappings, which generalize those introduced in [9] for the linear complementarity problem. Recall that $\Delta_{J}=\Delta_{J}(d) \doteq \operatorname{co}\left\{\frac{1}{d_{i}} e^{i}: i \in J\right\}$.

Definition 4.1. Let $d>0, \Phi: \mathbb{R}_{+}^{n} \hookrightarrow \mathbb{R}^{n}$ be a multifunction such that $0 \in \Phi(0)$. We say that $\Phi$ is a

- $\mathbf{T}(d)$-mapping, or $\Phi \in \mathbf{T}(d)$, if for any index subset $J \subseteq I$, one has

$$
\left.\begin{array}{l}
v \geq 0, w \geq 0, w \in \Phi(v) \\
w_{J}=0, \emptyset \neq \operatorname{supp}\{v\} \subseteq J
\end{array}\right\} \Longrightarrow v \in\left[\Phi\left(\operatorname{pos}^{+} \Delta_{J}\right)\right]^{*} .
$$

- $\tilde{\mathbf{T}}(d)$-mapping, or $\Phi \in \tilde{\mathbf{T}}(d)$, if for any index subset $J \subseteq I$, one has

$$
\left.\begin{array}{l}
v \geq 0, w \geq 0, w \in \Phi(v) \\
w_{J}=0, \emptyset \neq \operatorname{supp}\{v\} \subseteq J
\end{array}\right\} \Longrightarrow \begin{aligned}
& \langle y, x\rangle \geq 0 \\
& \forall x \in \operatorname{pos}^{+} \Delta_{J}, y \in \Phi(x)
\end{aligned}
$$

- $\mathbf{G T}(d)($ resp. $\mathbf{G} \tilde{\mathbf{T}}(d))$-mapping if it is $\mathbf{G}(d)$ and $\mathbf{T}(d)($ resp. $\tilde{\mathbf{T}}(d))$.

Remark 4.2. We observe that if $\Phi$ is $c$-subhomogeneous for some $c \in \mathcal{C}$, one obtains

$$
\begin{aligned}
& \Phi \in \mathbf{T}(d) \Longleftrightarrow \Phi \in \mathbf{T}\left(d^{\prime}\right) \forall d^{\prime}>0 \\
& \Phi \in \tilde{\mathbf{T}}(d) \Longleftrightarrow \Phi \in \tilde{\mathbf{T}}\left(d^{\prime}\right) \forall d^{\prime}>0 .
\end{aligned}
$$

Moreover, $\Phi \in \mathbf{T}(d)$ (resp. $\Phi \in \tilde{\mathbf{T}}(d)$ ) if and only if (4.1) with $\Delta_{J}$ instead of $\operatorname{pos}^{+} \Delta_{J}$ holds (resp. (4.2)).

Proposition 4.3. Let $d>0, c \in \mathcal{C}$, and $\Phi: \mathbb{R}_{+}^{n} \hookrightarrow \mathbb{R}^{n}$ be a multifunction. The following assertions hold:

(a) $0 \in \mathcal{S}(p, \Phi)$ for all $p \geq 0$ provided $0 \in \Phi(0)$;

(b) if $\Phi$ is copositive and $0 \in \Phi(0)$, then it is semimonotone (hence $\mathbf{G}(p)$ for all $p>0$ ) and $\tilde{\mathbf{T}}(p)$ for all $p>0$;

(c) if $\Phi$ is superadditive c-homogeneous (in particular if $\Phi$ is single-valued and linear) and $\tilde{\mathbf{T}}(d)$, then it is $\mathbf{T}(d)$.

Proof.

(a) It is obvious.

(b) For $p>0$ fixed, we take any $x \in \mathcal{S}(p, \Phi)$. Then, $y+p \geq 0$ and $\langle y+p, x\rangle=0$ for some $y \in \Phi(x)$. By copositivity $\langle p, x\rangle \leq 0$, which implies $x=0$, proving that $\Phi$ is semimonotone. The remaining assertion is obvious.

(c) Let $v, w$ be such that the left-hand side of (4.1) holds, then $\langle y, x\rangle \geq 0$ for all $x \in \operatorname{pos}^{+} \Delta_{J}$ and $y \in \Phi(x)$. By hypothesis, $y+c(t) w \in \Phi(x+t v)$ for all $t>0$ since $w \in \Phi(v)$. Thus $\langle y+c(t) w, x+t v\rangle \geq 0$ for all $t>0$. It follows that $\langle y, v\rangle \geq 0$ since $\langle w, x\rangle=\langle w, v\rangle=0$, and therefore (4.1) holds. 


\section{Example 4.4.}

- We must point to out that there is no relationship between $\mathbf{G}$ and $\mathbf{T}$-mappings, even in the linear case as shown in $[\mathrm{FL}]$. Analogously, there is no relationship between $\mathbf{G}$ and $\tilde{\mathbf{T}}$-mappings. Indeed, take

$$
M_{1}=\left(\begin{array}{cc}
-1 & 0 \\
0 & 1
\end{array}\right), M_{2}=\left(\begin{array}{cc}
0 & -1 \\
0 & 1
\end{array}\right) .
$$

Then, the mappings $\Phi_{i}(x)=M_{i} x, i=1,2$, satisfy $\Phi_{1} \in \tilde{\mathbf{T}}(d) \backslash \mathbf{G}(d)$ for any $d>0$, and $\Phi_{2} \in \mathbf{G}(d) \backslash \tilde{\mathbf{T}}(d)$ for any $d>0$.

- Let $M_{3}=\left(\begin{array}{cc}0 & -2 \\ 1 & 0\end{array}\right), M_{4}=\left(\begin{array}{cc}-1 & 0 \\ 0 & 0\end{array}\right)$, and consider the mappings $\Phi_{i}(x)=M_{i} x, i=3,4$. Then $\Phi_{3}$ is not copositive but it is semimonotone (hence $\Phi_{3} \in \mathbf{G}(p)$ for any $p>0$ ) and $\tilde{\mathbf{T}}(p)$ for any $p>0$; whereas $\Phi_{4} \in \mathbf{T}(p) \backslash \tilde{\mathbf{T}}(p)$ for any $p>0$. This shows that $\mathbf{T}$ and $\tilde{\mathbf{T}}$ does not coincide even in the linear case. The same properties hold for $\Phi_{i}(x)=\|x\| M_{i} x, i=3,4$.

One can check directly that

$$
\mathcal{F}(q, \Phi) \neq \emptyset \Longrightarrow\left[v \geq 0, v \in-\left[\Phi\left(\mathbb{R}_{+}^{n}\right)\right]^{*},\langle q, v\rangle \leq 0 \Longrightarrow\langle q, v\rangle=0\right] .
$$

The reverse implication holds whenever $\Phi$ is $c$-homogeneous for some $c \in \mathcal{C}, \Phi(0)=\{0\}$, and the set $\mathbb{R}_{+}^{n}-\Phi\left(\mathbb{R}_{+}^{n}\right)$ is convex and closed. It should be notice that the right-hand side of (4.3) amounts to writing

$$
v \geq 0, v \in-\left[\Phi\left(\mathbb{R}_{+}^{n}\right)\right]^{*} \Longrightarrow\langle q, v\rangle \geq 0
$$

Proposition 4.5. Let $d>0, c \in \mathcal{C}, q \in \mathbb{R}^{n}$, and $\Phi: \mathbb{R}_{+}^{n} \hookrightarrow \mathbb{R}^{n}$ be q-pseudomonotone c-homogeneous. Assume that $\mathcal{F}(q, \Phi) \neq \emptyset$. Then,

(a) $\Phi$ is copositive on $\mathbb{R}_{++}^{n}$;

(b) $\Phi$ is copositive, if in addition it is either lsc or superadditive;

(c) if $\Phi$ is superadditive, then for all $J \subseteq I$

$$
\left.\begin{array}{l}
v \geq 0, w \in \Phi(v), w_{J} \leq 0 \\
\emptyset \neq \operatorname{supp}\{v\} \subseteq J
\end{array}\right\} \Longrightarrow v \in\left[\Phi\left(\Delta_{J}\right)\right]^{*}
$$

Proof.

(a) Let $x^{0} \geq 0$ and $y^{0} \in \Phi\left(x^{0}\right)$ such that $y^{0}+q \geq 0$. For any $x>0$ there exists $t_{x}>0$ such that for all $t>t_{x}, \frac{t}{\|x\|_{d}} x-x^{0} \geq 0$. Thus $\left\langle y^{0}+q, \frac{t}{\|x\|_{d}} x-x^{0}\right\rangle \geq 0$ for all $t>t_{x}$. If $y \in \Phi(x)$, by $c$-homogeneity $\frac{c(t)}{c\left(\|x\|_{d}\right)} y \in \Phi\left(\frac{t}{\|x\|_{d}} x\right)$, and by $q$-pseudomonotonicity,

$$
\left\langle\frac{c(t)}{c\left(\|x\|_{d}\right)} y+q, t \frac{x}{\|x\|_{d}}-x^{0}\right\rangle \geq 0 \quad \forall t>t_{x}
$$

On dividing by $c(t) t$ and taking the limit as $t \rightarrow+\infty$, we get $\langle y, x\rangle \geq 0$, proving (a).

(b) Let $x$ be on the boundary of $\mathbb{R}_{+}^{n}$ and $y \in \Phi(x)$. Then, there exists $\left\{x^{k}\right\} \subseteq \mathbb{R}_{++}^{n}$ such that $x^{k} \rightarrow x$. Suppose first that $\Phi$ is lsc, then there exists $\left\{y^{k}\right\}$ such that $y^{k} \in \Phi\left(x^{k}\right)$ and $y^{k} \rightarrow y$. By (a), $\left\langle y^{k}, x^{k}\right\rangle \geq 0$ and then $\langle y, x\rangle \geq 0$. We now suppose that $\Phi$ is superadditive. If $e>0, t x+e>0$ for all $t>0$. Let $y \in \Phi(x)$ and $u \in \Phi(e)$. Clearly $c(t) y+u \in \Phi(t x+e)$, and by (a) $\langle c(t) y+u, t x+e\rangle \geq 0$ for all $t>0$. After dividing by $c(t) t$ and taking the limit as $t \rightarrow+\infty$, we get $\langle y, x\rangle \geq 0$. This completes the proof that $\Phi$ is copositive in either case. 
(c) Let $v, w$ be satisfy the left-hand side of (4.4). For $z \in \Delta_{J}$ and $y \in \Phi(z)$, we have $y+c(t) w \in \Phi(z+t v)$ for all $t>0$, and by (b) we get $\langle y+c(t) w, z+t v\rangle \geq 0$. Since $\langle w, v\rangle=\langle w, z\rangle \leq 0$, we deduce that $\langle y, v\rangle \geq 0$, proving (4.4).

The next result describes the asymptotic behavior of the normalized approximate solutions to problem (MCP), for the mappings introduced recently.

Lemma 4.6. Let $d>0, c \in \mathcal{C} ; \Phi, \Psi \in \mathcal{X}$, and $\left\{\sigma_{k}\right\}$ be an increasing sequence of positive numbers converging to $+\infty$. Assume there exist a sequence $\left\{\left(x^{k}, y^{k}+r^{k}\right)\right\} \in \mathcal{W}$ for $\Phi+\Psi$ such that $\frac{x^{k}}{\sigma_{k}} \rightarrow v$. Then, in addition to the existence of $w, r,\left\{w^{k}\right\}$ and subindex set $\emptyset \neq J_{v} \subseteq$ I satisfying the properties established in the basic lemma (for $\Phi^{k}=\Phi, \Psi^{k}=\Psi$, and $q^{k}=q$ for all $k$ ), we also obtain the following:

(a) for $\Phi$ to be c-subhomogeneous:

(a.1) $\Phi \in \mathbf{G}(d)$ and $\Psi$ uniformly bounded imply $w \geq 0, w_{J_{v}}=0$ (hence $\left.\langle w, v\rangle=0\right)$;

(a.2) $\Phi \in \mathbf{G} \tilde{\mathbf{T}}(d)$ and $\Psi$ copositive uniformly bounded imply $w \geq 0, w_{J_{v}}=0,\langle q, v\rangle \leq 0$, and $\langle y, x\rangle \geq 0$ $\forall x \in \operatorname{pos}^{+} \Delta_{J_{v}}$ and $y \in \Phi(x)$;

(a.3) $\Phi \in \mathbf{G T}(d)$ and $\Psi$ copositive zero-subhomogeneous imply $w \geq 0, w_{J_{v}}=0,\langle q, v\rangle \leq 0,\langle r, v\rangle \geq 0$, and $v \in\left[\Phi\left(\operatorname{pos}^{+} \Delta_{J_{v}}\right)\right]^{*}$;

(b) for $\Phi$ to be a copositive $c$-Moré and $\Psi$ copositive uniformly bounded: $\langle w, v\rangle=0$ and $\langle q, v\rangle \leq 0$.

Proof. We set $\Phi^{k}=\Phi, \Psi^{k}=\Psi$ and $q^{k}=q$ for all $k$ in the basic lemma.

(a.1) By (d) of the basic lemma, $\langle w, v\rangle \leq 0$. If, on the contrary $\langle w, v\rangle<0$, then from Remark $3.4,0 \neq v \in$ $\mathcal{S}(\tau d, \Phi)$ with $\tau=-\langle w, v\rangle>0$. This contradicts the fact that $\Phi \in \mathbf{G}(d)$. Hence $\langle w, v\rangle=0$. We then apply again (d) to obtain the desired result.

(a.2) If $\Phi \in \mathbf{G} \tilde{\mathbf{T}}(d)$, then (a.1) holds, and by (4.2) we get $\langle y, x\rangle \geq 0$ for all $x \in \operatorname{pos}^{+} \Delta_{J_{v}}$ and $y \in \Phi(x)$, which in turn implies $\left\langle y^{k_{m}}, x^{k_{m}}\right\rangle \geq 0$. From $\left\langle y^{k}+r^{k}+q, x^{k}\right\rangle \leq 0$ for all $k$ (set $x=0$ in $\left(\operatorname{MVIP}_{\mathrm{k}}\right)$ for $\left.\Phi+\Psi\right)$, we deduce that $\left\langle q, x^{k_{m}}\right\rangle \leq 0$, thus $\langle q, v\rangle \leq 0$.

(a.3) If $\Phi \in \mathbf{G T}(d)$, then (a.1) holds, and by (4.1) we get $v \in\left[\Phi\left(\operatorname{pos}^{+} \Delta_{J_{v}}\right)\right]^{*}$, which in turn implies $\left\langle y^{k_{m}}, v\right\rangle \geq$ 0 . From (c) of the basic lemma (for $z=v$ ), and setting $x=0$ in $\left(\mathrm{MVIP}_{\mathrm{k}}\right)$ for $\Phi+\Psi$ we get $\left\langle q+r^{k_{m}}, v\right\rangle \leq$ $\left\langle y^{k_{m}}+r^{k_{m}}+q, v\right\rangle=\left\langle y^{k_{m}}+r^{k_{m}}+q, \frac{x^{k_{m}}}{\sigma_{k_{m}}}\right\rangle \leq 0$, thus $\langle r+q, v\rangle \leq 0$. Since $\Psi$ is copositive $\langle r, v\rangle \geq 0$ and then $\langle q, v\rangle \leq 0$.

(b) By copositivity of $\Phi$ and $\Psi$, and (e) of the basic lemma, $\langle w, v\rangle=0$ and setting $x=0$ in $\left(\mathrm{MVIP}_{\mathrm{k}}\right)$ for $\Phi+\Psi,\left\langle q, x^{k}\right\rangle \leq\left\langle y^{k}+r^{k}+q, x^{k}\right\rangle \leq 0$. Thus $\langle q, v\rangle \leq 0$.

Given a nonempty set $C \subseteq \mathbb{R}^{n}$ and a vector $d>0$. We define the $d$-normalized asymptotic set of $C$ as the set

$$
C_{d}^{\infty} \doteq\left\{v \in \mathbb{R}^{n}: \exists x^{k} \in C,\left\|x^{k}\right\|_{d} \rightarrow+\infty, \frac{x^{k}}{\left\|x^{k}\right\|_{d}} \rightarrow v\right\},
$$

and the asymptotic cone of $C$ as the set

$$
C^{\infty} \doteq\left\{v \in \mathbb{R}^{n}: \exists x^{k} \in C, t_{k} \downarrow 0, t_{k} x^{k} \rightarrow v\right\}
$$

We notice that $C^{\infty}=\operatorname{pos} C_{d}^{\infty}$ (where by convention $\operatorname{pos} \emptyset=\{0\}$ ) and $C$ is bounded if and only if $C^{\infty}=\{0\}$, or equivalently, $C_{d}^{\infty}=\emptyset$ (see [3] for instance).

The following sets will be needed in our study:

$$
\begin{gathered}
\mathrm{U}_{q}(\Phi) \doteq\{v \geq 0: w \in \Phi(v),\langle w, v\rangle=0,\langle q, v\rangle \leq 0\}, \quad \mathrm{V}_{q}(\Phi) \doteq \mathbb{R}_{+}^{n} \cap\left[-\Phi\left(\mathbb{R}_{+}^{n}\right)-q\right]^{*} \\
\mathrm{~W}_{q}(\Phi) \doteq\{v \geq 0: w \in \Phi(v),\langle w, v\rangle=0, w \geq 0,\langle q, v\rangle \leq 0\}
\end{gathered}
$$


One immediately obtains

$$
q \in\left[\mathrm{U}_{0}(\Phi)\right]^{\#} \Longleftrightarrow \mathrm{U}_{q}(\Phi)=\{0\} ; \quad q \in[\mathcal{S}(0, \Phi)]^{\#} \Longleftrightarrow \mathrm{W}_{q}(\Phi)=\{0\}
$$

Proposition 4.7. Let $d>0, c \in \mathcal{C}, q \in \mathbb{R}^{n}$, and $\Phi, \Psi \in \mathcal{X}$.

(a) If $\Phi$ is c-subhomogeneous and $\Psi$ is uniformly bounded, $[\mathrm{S}(q, \Phi+\Psi)]_{d}^{\infty} \subseteq \mathcal{S}(0, \Phi) \cap \Delta_{d}$. If, in addition, $\Phi$ is either $\tilde{\mathbf{T}}(d)$ and $\Psi$ is copositive uniformly bounded or $\Phi$ is $\mathbf{T}(d)$ and $\Psi$ is copositive zero-subhomogeneous, $[\mathcal{S}(q, \Phi+\Psi)]_{d}^{\infty} \subseteq \mathrm{W}_{q}(\Phi) \cap \Delta_{d}$

(b) If $\Phi$ is copositive c-Moré and $\Psi$ is copositive uniformly bounded, $[\mathcal{S}(q, \Phi+\Psi)]_{d}^{\infty} \subseteq \mathrm{U}_{q}(\Phi) \cap \Delta_{d}$;

(c) If $\Phi$ is monotone and $\Psi$ is copositive zero-subhomogeneous, $[\mathcal{S}(q, \Phi+\Psi)]_{d}^{\infty} \subseteq \mathrm{V}_{q}(\Phi) \cap \Delta_{d}$. If, in addition, $\Phi$ is q-pseudomonotone, $[\mathcal{S}(q, \Phi)]^{\infty}=\mathrm{V}_{q}(\Phi)$ provided $\mathcal{S}(q, \Phi)$ is nonempty.

Proof.

(a) Let $v \in[\mathrm{S}(q, \Phi+\Psi)]_{d}^{\infty}$. Then, there exists $x^{k} \in \mathcal{S}(q, \Phi+\Psi)$ such that $\left\|x^{k}\right\|_{d} \rightarrow+\infty$ and $\frac{x^{k}}{\left\|x^{k}\right\|_{d}} \rightarrow v$. Moreover, there exist $y^{k} \in \Phi\left(x^{k}\right)$ and $r^{k} \in \Psi\left(x^{k}\right)$ such that $y^{k}+r^{k}+q \geq 0$ and $\left\langle y^{k}+r^{k}+q, x^{k}\right\rangle=0$ for all $k$. Clearly, $\sigma_{k}=\left\langle d, x^{k}\right\rangle \rightarrow+\infty$ and $\frac{x^{k}}{\sigma_{k}} \rightarrow v$ as $k \rightarrow+\infty$. Consequently, the basic lemma (for $\Phi^{k}=\Phi, \Psi^{k}=\Psi$, and $q^{k}=q$ for all $k$ ) implies the existence of $w \in \Phi(v)$ and $\emptyset \neq J_{v} \subseteq I$, such that (a)(d) of that lemma hold. Dividing $y^{k}+r^{k}+q \geq 0$ (resp. $\left\langle y^{k}+r^{k}+q, x^{k}\right\rangle=0$ ) by $c\left(\sigma_{k}\right)$ (resp. $\left.c\left(\sigma_{k}\right) \sigma_{k}\right)$ and taking the limit we obtain $w \geq 0,\langle w, v\rangle=0$, and $w_{J_{v}}=0$. Thus, in particular $v \in \mathcal{S}(0, \Phi)$.

Let $\Phi$ be $\mathbf{T}(d)$ (resp. $\tilde{\mathbf{T}}(d)$ ) and $\Psi$ be zero-subhomogeneous (resp. uniformly bounded) copositive, by proceeding exactly as in Lemma 4.6 we obtain that $\langle q, v\rangle \leq 0$. Thus, $v \in \mathrm{W}_{q}(\Phi)$.

(b) By proceeding as above and in Lemma 4.6, we obtain that $v \in \mathrm{U}_{q}(\Phi)$.

(c) If $\Phi$ is monotone and $\Psi$ is zero-subhomogeneous, by proceeding as above, (g) of the basic lemma implies that $v \in \mathrm{V}_{q}(\Phi)$.

If $\Phi$ is $q$-pseudomonotone, it is well known that (see [5] for instance)

$$
\mathcal{S}(q, \Phi)=\bigcap_{x \geq 0} \bigcap_{y \in \Phi(x)}\{\bar{x} \geq 0:\langle y+q, x-\bar{x}\rangle \geq 0\} .
$$

Since the sets involved in the intersection are closed and convex and $\mathcal{S}(q, \Phi)$ is nonempty, applying a property of asymptotic cones we conclude that

$$
[\mathcal{S}(q, \Phi)]^{\infty}=\bigcap_{x \geq 0} \bigcap_{y \in \Phi(x)}\{\bar{x} \geq 0:\langle y+q, x-\bar{x}\rangle \geq 0\}^{\infty}=\mathrm{V}_{q}(\Phi)
$$

since $\{\bar{x} \geq 0:\langle y+q, x-\bar{x}\rangle \geq 0\}^{\infty}=\{v \geq 0:\langle y+q, v\rangle \leq 0\}$.

Example 4.8. The inclusions in the preceding proposition may be strict.

- Let $\Phi\left(x_{1}, x_{2}\right)=\left(\begin{array}{ll}0 & 1 \\ 0 & 1\end{array}\right)\left(\begin{array}{l}x_{1} \\ x_{2}\end{array}\right)$ be in $\mathbf{T}(d) \cap \tilde{\mathbf{T}}(d)$ (for all $\left.d>0\right), \Psi=0$, and $q=\left(-\frac{1}{2},-1\right)^{\mathrm{T}}$. Since $\mathcal{S}(q, \Phi)=\left\{(0,1)^{\mathrm{T}}\right\}$ and $\mathcal{S}(0, \Phi)=\mathrm{W}_{q}(\Phi)=\left\{\left(v_{1}, 0\right)^{\mathrm{T}}: v_{1} \geq 0\right\}$, the inclusions in $(a)$ are strict.

- Let $\Phi\left(x_{1}, x_{2}\right)=\left[x_{1}, 2 x_{1}\right] \times\{0\}$ be copositive $\frac{\lambda}{2}$-Moré on $\Delta_{d}$ for $d=(1,1)^{\mathrm{T}}, \Psi=0$, and $q=(-1,1)^{\mathrm{T}}$. Since $\mathcal{S}(q, \Phi)=\left[\frac{1}{2}, 1\right] \times\{0\}$ and $\mathrm{U}_{0}(\Phi)=\left\{\left(0, v_{2}\right)^{\mathrm{T}}: v_{2} \geq 0\right\}$, the inclusion in $(b)$ is strict. 


\section{Main existence Results}

In this section we present new existence results, which generalize and unify several ones found in the literature. This is carried out by using the classes of mappings introduced in Sections 2 and 4 applying mostly Theorem 3.1. Actually, our main results of this section establishes sufficient conditions implying a kind of robustness property for some classes mappings with respect to certain perturbation.

Lemma 5.1. Let $d>0, c \in \mathcal{C}, q \in \mathbb{R}^{n}$, and $\Phi, \Psi \in \mathcal{X}$. The set $\mathcal{D}(\Phi+\Psi)$ is closed at $q$ under any of the following circumstances:

(a) $\Phi$ is c-subhomogeneous $\mathbf{G T}(d), \Psi$ is copositive zero-subhomogeneous, and $q \in[\mathcal{S}(0, \Phi)]^{\#}$;

(b) $\Phi$ is c-subhomogeneous $\mathbf{G} \tilde{\mathbf{T}}(d), \Psi$ is copositive uniformly bounded, and $q \in[\mathcal{S}(0, \Phi)]^{\#}$;

(c) $\Phi$ is copositive c-Moré, $\Psi$ is copositive uniformly bounded, and $q \in\left[\mathrm{U}_{0}(\Phi)\right]^{\#}$;

(d) $\Phi$ is monotone, $\Psi$ is copositive zero-subhomogeneous, and $V_{q}(\Phi)=\{0\}$.

Proof. Let $\left\{q^{k}\right\} \subseteq \mathcal{D}(\Phi+\Psi)$ be a sequence converging to $q$. There exist $x^{k} \geq 0, y^{k} \in \Phi\left(x^{k}\right)$, and $r^{k} \in \Psi\left(x^{k}\right)$ such that $y^{k}+r^{k}+q^{k} \geq 0$ and $\left\langle y^{k}+r^{k}+q^{k}, x^{k}\right\rangle=0$. If the sequence $\left\{x^{k}\right\}$ is bounded, each of its limit points is in $\mathcal{S}(q, \Phi+\Psi)$ since $\Phi, \Psi \in \mathcal{X}$. Thus $q \in \mathcal{D}(\Phi+\Psi)$.

If the sequence $\left\{x^{k}\right\}$ is unbounded, setting $\sigma_{k}=\left\langle d, x^{k}\right\rangle \rightarrow+\infty$, we may consider that there exists $v$ such that, up to subsequences, $\frac{x^{k}}{\sigma_{k}} \rightarrow v$ and $\left\{\left(x^{k}, y^{k}, r^{k}\right)\right\}$ are solutions to $\left(\mathrm{PMVIP}_{\mathrm{k}}\right)$ for $\Phi^{k}=\Phi, \Psi^{k}=\Psi$ for all $k$. By the basic lemma (for $\Phi^{k}=\Phi, \Psi^{k}=\Psi$ for all $k$ ) and proceeding as in Lemma 4.6 it follows that:

(a) there exist $\left\{x^{k_{m}}\right\}, r$, and $\emptyset \neq J_{v} \subseteq I$, such that $0 \neq v \in \mathcal{S}(0, \Phi), w_{J_{v}}=0$, and by (4.1) $v \in$ $\left[\Phi\left(\operatorname{pos}^{+} \Delta_{J_{v}}\right)\right]^{*}$, which in turn implies $\left\langle y^{k_{m}}, v\right\rangle \geq 0$. Moreover, from $(c)$ of the basic lemma (for $\left.z=v\right)$ we get $\left\langle y^{k_{m}}+r^{k_{m}}+q^{k_{m}}, v\right\rangle=\left\langle y^{k_{m}}+r^{k_{m}}+q^{k_{m}}, \frac{x^{k_{m}}}{\sigma_{k_{m}}}\right\rangle=0$, thus $\left\langle r^{k_{m}}+q^{k_{m}}, v\right\rangle \leq 0$, then $\langle q, v\rangle \leq$ $\langle r+q, v\rangle \leq 0$, contradicting the choice of $q$

(b) there exist $\left\{x^{k_{m}}\right\}$ and $\emptyset \neq J_{v} \subseteq I$, such that $0 \neq v \in \mathcal{S}(0, \Phi), w_{J_{v}}=0$, and $\langle y, x\rangle \geq 0$ for all $x \in$ $\operatorname{pos}^{+} \Delta_{J_{v}}, y \in \Phi(x)$, which in turn implies $\left\langle y^{k_{m}}, x^{k_{m}}\right\rangle \geq 0$. Moreover, from $\left\langle y^{k_{m}}+r^{k_{m}}+q^{k_{m}}, x^{k_{m}}\right\rangle=0$, we get $\left\langle q^{k_{m}}, x^{k_{m}}\right\rangle \leq 0$, then $\langle q, v\rangle \leq 0$, contradicting the choice of $q$;

(c) there exists $w^{k} \in \Phi\left(\frac{x^{k}}{\sigma_{k}}\right)$ such that $w^{k} \rightarrow w \in \Phi(v)$ and $\langle w, v\rangle=0$, thus $0 \neq v \in \mathrm{U}_{0}(\Phi)$. Moreover, from $0=\left\langle y^{k}+r^{k}+q^{k}, x^{k}\right\rangle \geq\left\langle q^{k}, x^{k}\right\rangle$, we get $\langle q, v\rangle \leq 0$, contradicting the choice of $q$

(d) $0 \neq v \in V_{q}(\Phi)$ a contradiction.

We first obtain existence theorems for problem (MCP) for mappings of the form $\Phi+\Psi$, and $\Phi$ respectively. In this way, we generalize some results from $[13,20-22]$ as will be shown in the example below.

Theorem 5.2. Let $d>0, c \in \mathcal{C}, \Phi \in \mathcal{X}$ be $\mathbf{G T}(d)$ (resp. $\mathbf{G} \tilde{\mathbf{T}}(d)$ ) c-subhomogeneous, and $\Psi \in \mathcal{X}$ be a zero-subhomogeneous (resp. uniformly bounded) copositive mapping:

(a) if $q \in[\mathcal{S}(0, \Phi)]^{*}$ and $\mathcal{D}(\Phi+\Psi)$ is closed at $q$, then $\mathcal{S}(q, \Phi+\Psi)$ is nonempty;

(b) if $q \in[\mathcal{S}(0, \Phi)]^{\#}$, then $\mathcal{S}(q, \Phi+\Psi)$ is nonempty and compact. 
Proof.

(a) Let $\left\{\sigma_{k}\right\}$ be an increasing sequence of positive numbers converging to $+\infty, d>0$, and $\left\{\left(x^{k}, y^{k}+r^{k}\right)\right\} \in \mathcal{W}$ for $\Phi+\Psi$. Since $\left\langle d, \frac{x^{k}}{\sigma_{k}}\right\rangle=1$, up to subsequences, there exists $0 \neq v \geq 0$ such that $\frac{x^{k}}{\sigma_{k}} \rightarrow v$. Thus,

$$
x^{k} \in D_{k}, y^{k} \in \Phi\left(x^{k}\right), r^{k} \in \Psi\left(x^{k}\right),\left\langle y^{k}+r^{k}+q, x-x^{k}\right\rangle \geq 0 \quad \text { for all } x \in D_{k}
$$

By the basic lemma (for $\Phi^{k}=\Phi, \Psi^{k}=\Psi$ and $q^{k}=q$ for all $k$ ), Lemma 4.6 for $\Phi$ in $\mathbf{G} \tilde{\mathbf{T}}(d)$ (resp. in GT $(d)$ ) and the uniform boundedness (resp. zero-subhomogeneity) and the copositivity of $\Psi$, there exist $w \in \Phi(v), r$, $\emptyset \neq J_{v} \subseteq I$, and $\left\{x^{k_{m}}\right\}$ such that $w \geq 0,\langle w, v\rangle=0, w_{J_{v}}=0,\langle q, v\rangle \leq 0$, and $\langle y, x\rangle \geq 0$ for all $x \in \operatorname{pos}^{+} \Delta_{J_{v}}$, $y \in \Phi(x)$ (resp. $v \in\left[\Phi\left(\operatorname{pos}^{+} \Delta_{J_{v}}\right)\right]^{*}, r \in \Psi(v)$, and $\left.\langle r, v\rangle \geq 0\right)$, which in turn implies $\left\langle y^{k_{m}}, x^{k_{m}}\right\rangle \geq 0$ (resp. $\left.\left\langle y^{k_{m}}, v\right\rangle \geq 0\right)$. Moreover, $v \in \mathcal{S}(0, \Phi)$ implies $\langle q, v\rangle=0$. From (3.4) for $\Phi+\Psi$, we get

$$
\theta_{k_{m}}=-\left\langle y^{k_{m}}+r^{k_{m}}+q, \frac{x^{k_{m}}}{\sigma_{k_{m}}}\right\rangle=-\left\langle y^{k_{m}}+r^{k_{m}}+q, v\right\rangle \text {. }
$$

Then $0 \leq \theta_{k_{m}} \leq-\left\langle q, \frac{x^{k_{m}}}{\sigma_{k_{m}}}\right\rangle$ (resp. $0 \leq \theta_{k_{m}} \leq-\left\langle r^{k_{m}}, v\right\rangle$ ). Thus $\liminf _{k \rightarrow+\infty} \theta_{k}=0$, and the result follows from Theorem 3.1.

(b) By Lemma 5.1 the set $\mathcal{D}(\Phi+\Psi)$ is closed at $q$ and by (a) we conclude that $\mathcal{S}(q, \Phi+\Psi)$ is nonempty. Its boundedness follows from Proposition 4.7 since by the choice of $q, \mathrm{~W}_{q}(\Phi)=\{0\}$.

When $\Psi=0$ in the previous theorem, the closedness of $\mathcal{D}(\Phi)$ is not needed in some cases, as shown by the next corollary.

Corollary 5.3. Let $d>0, c \in \mathcal{C}, q \in \mathbb{R}^{n}$, and $\Phi \in \mathcal{X}$ be c-subhomogeneous:

(a) if $\Phi \in \mathbf{G T}(d)$ and $q \in[\mathcal{S}(0, \Phi)]^{*}$, then $\mathcal{S}(q, \Phi)$ is nonempty;

(b) if $\Phi \in \mathbf{G} \tilde{\mathbf{T}}(d), \mathcal{D}(\Phi)$ is closed at $q$, and $q \in[\mathcal{S}(0, \Phi)]^{*}$, then $\mathcal{S}(q, \Phi)$ is nonempty;

(c) if $\Phi$ is $\mathbf{G T}(d)$ or $\mathbf{G} \tilde{\mathbf{T}}(d)$ and $q \in[\mathcal{S}(0, \Phi)]^{\#}$, then $\mathcal{S}(q, \Phi)$ is nonempty and compact.

Proof. By setting $\Psi=0$ in the above theorem we obtain (b) and (c).

By proceeding exactly as in the above theorem with $\Psi=0$, from (5.2) we obtain that $0 \leq \theta_{k_{m}} \leq$ $-\left\langle y^{k_{m}}+q, v\right\rangle \leq 0$, thus $\theta_{k_{m}}=0$ and $x^{k_{m}} \in \mathcal{S}(q, \Phi)$.

Remark 5.4. Since copositive mappings are $\mathbf{G} \tilde{\mathbf{T}}(d)$ for each $d>0$, the above result contains Corollary 2 of $[13]$.

The next two results do not require $c$-subhomogeneity.

Theorem 5.5. Let $d>0, c \in \mathcal{C}, q \in \mathbb{R}^{n}, \Phi \in \mathcal{X}$ be copositive c-Moré, and $\Psi \in \mathcal{X}$ be copositive uniformly bounded:

(a) if $q \in\left[\mathrm{U}_{0}(\Phi)\right]^{*}$ and $\mathcal{D}(\Phi+\Psi)$ is closed at $q$, then $\mathcal{S}(q, \Phi+\Psi)$ is nonempty;

(b) if $q \in\left[\mathrm{U}_{0}(\Phi)\right]^{\#}$, then $\mathcal{S}(q, \Phi+\Psi)$ is nonempty and compact.

Proof.

(a) Let $\left\{\sigma_{k}\right\}$ be an increasing sequence of positive numbers converging to $+\infty, d>0$, and $\left\{\left(x^{k}, y^{k}\right)\right\} \in \mathcal{W}$ for $\Phi+\Psi$. Since $\left\langle d, \frac{x^{k}}{\sigma_{k}}\right\rangle=1$, up to subsequences, there exists $0 \neq v \geq 0$ such that $\frac{x^{k}}{\sigma_{k}} \rightarrow v$. By the basic lemma (for $\Phi^{k}=\Phi, \Psi^{k}=\Psi$, and $q^{k}=q$ for all $k$ ) and Lemma 4.6 there exist $w, r,\left\{x^{k_{m}}\right\}$, and $\emptyset \neq J_{v} \subseteq I$ such that $w \in \Phi(v)$ and $\langle w, v\rangle=0$, thus $v \in \mathrm{U}_{0}(\Phi)$, and then $\langle q, v\rangle \geq 0$. From (5.2) and copositivity of $\Phi$ and $\Psi$ we get that $0 \leq \theta_{k_{m}}=-\left\langle y^{k_{m}}+r^{k_{m}}+q, \frac{x^{k_{m}}}{\sigma_{k_{m}}}\right\rangle \leq-\left\langle q, \frac{x^{k_{m}}}{\sigma_{k_{m}}}\right\rangle$, thus $\liminf _{k \rightarrow \infty} \theta_{k}=0$ and the result follows from Theorem 3.1. 
(b) By Lemma 5.1 the set $\mathcal{D}(\Phi+\Psi)$ is closed at $q$, and by (a) we conclude that $\mathcal{S}(q, \Phi+\Psi)$ is nonempty. Its boundedness follows from Proposition 4.7 , since by the choice of $q, \mathrm{U}_{q}(\Phi)=\{0\}$.

The previous theorem allows us to recover Theorem 3.1 from [24], where $\Phi$ is assumed to admit contractible images.

Corollary 5.6. Let $\Phi \in \mathcal{X}$ be such that $0 \in \Phi(0)$. If $\Phi$ is strongly copositive, then $\mathcal{S}(q, \Phi)$ is nonempty and compact for all $q \in \mathbb{R}^{n}$.

Proof. By Proposition 2.5, $\Phi$ is $c$-Moré for some $c \in \mathcal{C}$, moreover $\mathrm{U}_{0}(\Phi)=\{0\}$. The result follows from the above theorem.

Theorem 5.7. Let $d>0, q \in \mathbb{R}^{n}, \Phi \in \mathcal{X}$ be monotone copositive, and $\Psi \in \mathcal{X}$ be copositive zero-subhomogeneous:

(a) if the following implication holds $\left[v \in \mathrm{V}_{q}(\Phi) \Longrightarrow\langle q, v\rangle=0\right]$ and $\mathcal{D}(\Phi+\Psi)$ is closed at $q$, then $\mathcal{S}(q, \Phi+\Psi)$ is nonempty;

(b) if $\mathrm{V}_{q}(\Phi)=\{0\}$, then $\mathcal{S}(q, \Phi+\Psi)$ is nonempty and compact.

Proof.

(a) Let $\left\{\sigma_{k}\right\}$ be an increasing sequence of positive numbers converging to $+\infty, d>0$, and $\left\{\left(x^{k}, y^{k}+r^{k}\right)\right\} \in$ $\mathcal{W}$ for $\Phi+\Psi$. Since $\left\langle d, \frac{x^{k}}{\sigma_{k}}\right\rangle=1$, up to subsequences, there exists $0 \neq v \geq 0$ such that $\frac{x^{k}}{\sigma_{k}} \rightarrow v$. By $(\mathrm{g})$ of the basic lemma (for $\Phi^{k}=\Phi, \Psi^{k}=\Psi$, and $q^{k}=q$ for all $k$ ), $v \in \mathrm{V}_{q}(\Phi)$ and by hypothesis $\langle q, v\rangle=0$. From (5.2) since $\Phi$ and $\Psi$ are copositive we get $0 \leq \theta_{k_{m}}=-\left\langle y^{k_{m}}+r^{k_{m}}+q, \frac{x^{k_{m}}}{\sigma_{k_{m}}}\right\rangle \leq-\left\langle q, \frac{x^{k_{m}}}{\sigma_{k_{m}}}\right\rangle$. Therefore $\liminf _{k \rightarrow+\infty} \theta_{k}=0$ and the result follows from Theorem 3.1.

(b) By Lemma 5.1 the set $\mathcal{D}(\Phi+\Psi)$ is closed at $q$ and by (a) we conclude that $\mathcal{S}(q, \Phi+\Psi)$ is nonempty. Its boundedness follows from Proposition 4.7, since $\mathrm{V}_{q}(\Phi)=\{0\}$.

It is worth mentioning that a monotone mapping is copositive if $\Phi(0) \cap \mathbb{R}_{+}^{n} \neq \emptyset$ (in particular if $0 \in \Phi(0)$ ).

We now revise the pseudomonotone case. Part of the next theorem was first observed in [5], see also [8].

Theorem 5.8. Let $q \in \mathbb{R}^{n}$ and $\Phi \in \mathcal{X}$ be q-pseudomonotone. Consider the statements

(a) $\mathcal{F}_{s}(q, \Phi)$ is nonempty;

(b) $\mathrm{V}_{q}(\Phi)=\{0\}$;

(c) $\mathcal{S}(q, \Phi)$ is nonempty and compact;

(d) There exists a compact convex set $K \subseteq \mathbb{R}_{+}^{n}$ such that

$$
\forall x \in \mathbb{R}_{+}^{n} \backslash K \forall y \in \Phi(x) \exists z \in K:\langle y+q, z-x\rangle<0 .
$$

The following implications hold: $\quad(\mathrm{a}) \Longrightarrow(\mathrm{b}) \Longleftrightarrow(\mathrm{c}) \Longleftrightarrow(\mathrm{d})$.

Moreover, if $\Phi\left(\mathbb{R}_{+}^{n}\right)$ is convex, then all the statements are equivalent.

Proof. $(\mathrm{a}) \Rightarrow(\mathrm{b})$ : Let $x^{0} \geq 0$ and $y^{0} \in \Phi\left(x^{0}\right)$ such that $y^{0}+q>0$, and let $v \in \mathrm{V}_{q}(\Phi)$, thus $\left\langle y^{0}+q, v\right\rangle \leq 0$ a contradiction if $v \neq 0$.

(b) $\Rightarrow(\mathrm{c})$ : Let $\left\{\sigma_{k}\right\}$ be an increasing sequence of positive numbers converging to $+\infty, d>0$, and $\left\{\left(x^{k}, y^{k}, \theta_{k}\right)\right\}$ a sequence which solves $\left(\mathrm{MCP}_{\mathrm{k}}\right)$ for all $k$. If there exists $k$ such that $\left\langle d, x^{k}\right\rangle<\sigma_{k}$, then $\theta_{k}=0$ and therefore $x^{k} \in \mathcal{S}(q, \Phi)$. If $\left\langle d, x^{k}\right\rangle=\sigma_{k}$ for all $k$, then up to subsequences $\frac{x^{k}}{\sigma_{k}} \rightarrow v \neq 0$. By (f) of the basic lemma (for $\Phi^{k}=\Phi, \Psi^{k}=0$, and $q^{k}=q$ for all $k$ ) we obtain $0 \neq v \in \mathrm{V}_{q}(\Phi)$ a contradiction. The boundedness of the solution set follows from Proposition 4.7.

$(\mathrm{c}) \Rightarrow(\mathrm{b})$ : It follows from (c) of Proposition 4.7 .

$(\mathrm{c}) \Rightarrow(\mathrm{d})$ : See $[6]$.

$(\mathrm{d}) \Rightarrow(\mathrm{c})$ : See [5] (this implication holds without the $q$-pseudomonotonicity assumption).

(b) $\Rightarrow(\mathrm{a})$ : On the contrary suppose that $\left(\Phi\left(\mathbb{R}_{+}^{n}\right)+q\right) \cap \operatorname{int} \mathbb{R}_{+}^{n}=\emptyset$. By using standard separation arguments, we obtain the existence of $0 \neq v \in \mathrm{V}_{q}(\Phi)$ a contradiction. 
Theorem 5.9. Let $d>0, c \in \mathcal{C}, q \in \mathbb{R}^{n}$, and $\Phi \in \mathcal{X}$ be $q$-pseudomonotone $c$-homogeneous and superadditive. Consider the statements:

(a) $\mathcal{F}(q, \Phi) \neq \emptyset$;

(b) $v \geq 0, v \in-\left[\Phi\left(\mathbb{R}_{+}^{n}\right)\right]^{*} \Longrightarrow\langle q, v\rangle \geq 0$;

(c) $\mathcal{S}(q, \Phi) \neq \emptyset$.

The following implications hold: $\quad(\mathrm{c}) \Longleftrightarrow(\mathrm{a}) \Longrightarrow(\mathrm{b})$.

Moreover, if $\mathbb{R}_{+}^{n}-\Phi\left(\mathbb{R}_{+}^{n}\right)$ is convex and closed together with $\Phi(0)=\{0\}$, then all the statements are equivalent.

Proof. $(\mathrm{a}) \Rightarrow(\mathrm{b})$ : It follows from (4.3).

$(\mathrm{a}) \Rightarrow(\mathrm{c})$ : Let $\left\{\sigma_{k}\right\}$ be an increasing sequence of positive numbers converging to $+\infty, d>0$, and $\left\{\left(x^{k}, y^{k}\right)\right\} \in \mathcal{W}$. Since $\left\langle d, \frac{x^{k}}{\sigma_{k}}\right\rangle=1$, up to subsequences, there exists $0 \neq v \geq 0$ such that $\frac{x^{k}}{\sigma_{k}} \rightarrow v$. By (f) of the basic lemma (for $\Phi^{k}=\Phi, \Psi^{k}=0$, and $q^{k}=q$ for all $k$ ) we get $0 \leq v \in-\left[\Phi\left(\mathbb{R}_{+}^{n}\right)\right]^{*}$ and $\langle q, v\rangle \leq 0$, which in turn imply $\langle q, v\rangle=0$ by the above implication. By $(\mathrm{d})$ of the same lemma and (4.4) we conclude that $v \in\left[\Phi\left(\Delta_{J_{v}}\right)\right]^{*}$, which in turn implies $\left\langle y^{k_{m}}, v\right\rangle \geq 0$, therefore by $(3.4), 0 \leq \theta_{k_{m}}=-\left\langle y^{k_{m}}+q, v\right\rangle=-\left\langle y^{k_{m}}, v\right\rangle \leq 0$, thus $\theta_{k_{m}}=0$ and then $x^{k_{m}} \in \mathcal{S}(q, \Phi)$.

$(\mathrm{c}) \Rightarrow(\mathrm{a})$ : It is obvious.

$(\mathrm{b}) \Rightarrow(\mathrm{a})$ : It follows from the remark made after $(4.3)$.

In what follows we give a variety of results existing in the literature which are direct consequences of our theorems.

Example 5.10.

1. [13], Corollary 4,5, let $\Phi(x)=M x$, where $M \in \mathbb{R}^{n \times n}$ and $\Psi(x)=\partial h(x)$, where $h$ is a nonnegative on $\mathbb{R}_{+}^{n}$ support function of a nonempty compact convex set $C$ (see Ex. 2.2). By applying Theorem 5.2 we obtain that:

- if $M$ is copositive, $q \in[\mathcal{S}(0, M)]^{*}$, and $\mathcal{D}(M+\partial h)$ is closed at $q$, then $\mathcal{S}(q, M+\partial h)$ is nonempty. Moreover, if $C$ is a polyhedral set the closedness condition is clearly satisfied [13], Proposition 3;

- if $M$ is copositive-star and there exists a vector $x^{0} \geq 0$ such that $M x^{0}+q>0$, then $\mathcal{S}(q, M+\partial h)$ is nonempty and compact. Since the existence of such an $x^{0}$ implies that $q \in[\mathcal{S}(0, M)]^{\#}$;

- if $M$ is regular, then $\mathcal{S}(q, M+\partial h)$ is nonempty and compact for all $q \in \mathbb{R}^{n}$.

2. Let $\Phi(x)=F(x)$, where $F: \mathbb{R}_{+}^{n} \rightarrow \mathbb{R}^{n}$ is a continuous homogeneous of degree $\gamma>0$ function. Let $\Psi: \mathbb{R}_{+}^{n} \hookrightarrow \mathbb{R}^{n}$ be a multifunction.

- [22], Theorem 7, if $F$ is regular and $\Psi$ be an usc convex-valued uniformly bounded multifunction, then $\mathcal{S}(q, F+\Psi)$ is nonempty and compact for all $q \in \mathbb{R}^{n}$. This follows from Theorem 5.2;

- [21], Theorem 3.3, if $F$ is monotone, $\partial h(x)$ as above, and there exist $u \geq 0, \tilde{y} \in \partial h(u)$ such that $F(u)+\tilde{y}+q>$ 0 , then $\mathcal{S}(q, F+\partial h)$ is nonempty and compact. This follows from Theorem 5.8 since $F+\partial h$ is $q$-pseudomonotone and $\mathcal{F}_{s}(q, F+\partial h)$ is nonempty.

Remark 5.11. The results of this section allow us to find Karush-Kuhn-Tucker stationary points for the following mathematical programming problem:

$$
\begin{array}{ll}
\text { minimize } & F(x)+h(x) \\
\text { subject to } & x \geq 0, g(x) \geq 0
\end{array}
$$

where $F: \mathbb{R}^{n} \rightarrow \mathbb{R}$ and $g: \mathbb{R}^{n} \rightarrow \mathbb{R}^{m}$ are differentiable functions and $h$ is the support function of a nonempty compact convex set in $\mathbb{R}^{n}$, since its corresponding Karush-Kuhn-Tucker stationary point problem can be expressed as a multivalued complementarity problem [21].

Given $d>0$, the system

$$
v \geq 0,\langle d, v\rangle=1, w \in \Phi(v),\langle w, v\rangle \leq 0, w-\langle w, v\rangle d \geq 0
$$


found in the basic lemma (for $\Phi^{k}=\Phi, \Psi^{k}=\Psi$, and $q^{k}=q$ for all $k$ ), plays a fundamental role in characterizing the nonemptiness and boundedness of $\mathcal{S}(q, \Phi)$ for all $q \in \mathbb{R}^{n}$. When $\Phi$ is $c$-subhomogeneous the inconsistency of (5.3) is equivalent to the inconsistency of the following system

$$
0 \neq v \geq 0, z \in \Phi(v), \tau \geq 0, z+\tau d \geq 0,\langle z+\tau d, v\rangle=0 .
$$

This system has its origin in [15] where the case $\Phi(x)=M x$ with $M$ being a matrix and $d$ to be the vector of ones is treated. It was further developed in [16] for $\Phi$ having single-values and nonlinear. Afterwards, the set-valued version was introduced in [13,22].

The next theorem generalizes Corollary 2 of [13] and provides, in this setting, new characterizations of regular mappings. In particular, it shows the existence of some kind of robustness property with respect to certain classes of perturbations.

Theorem 5.12. Let $d>0, c \in \mathcal{C}$, and $\Phi \in \mathcal{X}$ be c-subhomogeneous. Consider the statements

(a) the system (5.3) is inconsistent;

(b) $\Phi \in \mathbf{G}(d)$ and $\mathcal{S}(q, \Phi+\Psi)$ is nonempty and compact for all $q \in \mathbb{R}^{n}$ and all $\Psi \in \mathcal{X}$ copositive uniformly bounded;

(c) $\Phi \in \mathbf{G}(d)$ and $\mathcal{S}(q, \Phi+\Psi)$ is nonempty and compact for all $q \in \mathbb{R}^{n}$ and all $\Psi \in \mathcal{X}$ copositive zerosubhomogeneous;

(d) $\Phi \in \mathbf{G}(d)$ and $\mathcal{S}(q, \Phi)$ is nonempty and compact for all $q \in \mathbb{R}^{n}$;

(e) $\Phi \in \mathbf{R}(d)$.

The following implications hold: $(\mathrm{e}) \Longleftrightarrow(\mathrm{a}) \Longrightarrow(\mathrm{b}) \Longrightarrow(\mathrm{c}) \Longrightarrow(\mathrm{d})$. Moreover, if $\Phi$ is c-homogeneous, then all the statements are equivalent.

Proof. (a) $\Rightarrow(\mathrm{b})$ : We first prove that $\Phi$ is $\mathbf{G}(d)$. Let $\tau>0$ and $x \in \mathcal{S}(\tau d, \Phi)$. Then there is $y \in \Phi(x)$ such that $y+\tau d \geq 0$ and $\langle y+\tau d, x\rangle=0$. If $\langle y, x\rangle=0$ then $\langle d, x\rangle=0$, which implies $x=0$. If $\langle y, x\rangle<0$ then for $v=x /\|x\|_{d}$ we get $w=y / c\left(\|x\|_{d}\right) \in \Phi(v)$ and since $\tau\|x\|_{d}=-\langle y, x\rangle$, clearly (5.3) holds, a contradiction. The previous reasoning also shows that $\mathcal{S}(0, \Phi)=\{0\}$, and thus $\Phi \in \tilde{\mathbf{T}}(d)$. Hence $\Phi \in \mathbf{G} \tilde{\mathbf{T}}(d)$, and by Theorem 5.2 we conclude that $\mathcal{S}(q, \Phi+\Psi)$ is nonempty and compact for all $q \in \mathbb{R}^{n}$ and all $\Psi \in \mathcal{X}$ copositive uniformly bounded.

$(\mathrm{a}) \Leftrightarrow(\mathrm{e})$ : It follows from the equivalence between (5.3) and (5.4).

(b) $\Rightarrow$ (c): It follows from Proposition 2.3(b).

$(\mathrm{c}) \Rightarrow(\mathrm{d})$ : It is obvious.

$(\mathrm{d}) \Rightarrow(\mathrm{e})$ : If there exists $v \in \mathcal{S}(0, \Phi), v \neq 0$, then by $c$-homogeneity, $t v \in \mathcal{S}(0, \Phi)$ for all $t>0$, contradicting the boundedness of $\mathcal{S}(0, \Phi)$.

We rewrite the previous theorem to get the next corollary which is new in the literature, even in the case when $\Phi(x)=M x$ with $M$ being a real matrix. Our corollary gives more information than the existing ones, e.g. [13].

Corollary 5.13. Let $d>0, c \in \mathcal{C}$, and $\Phi \in \mathcal{X}$ be c-homogeneous. Assume in addition that $\Phi \in \mathbf{G}(d)$. The following assertions are equivalent:

(a) $\mathcal{S}(q, \Phi)$ is nonempty and compact for all $q \in \mathbb{R}^{n}$;

(b) $\mathcal{S}(q, \Phi+\Psi)$ is nonempty and compact for all $q \in \mathbb{R}^{n}$ and all $\Psi \in \mathcal{X}$ copositive uniformly bounded;

(c) $\mathcal{S}(q, \Phi+\Psi)$ is nonempty and compact for all $q \in \mathbb{R}^{n}$ and all $\Psi \in \mathcal{X}$ copositive zero-subhomogeneous;

(d) $\mathcal{S}(0, \Phi)=\{0\}$.

\section{Sensitivity, stability Results, and approximable mappings}

In this section we give sensitivity results for problem (MCP), whose data are small perturbations of a given pair $\left(q^{0}, \Phi^{0}\right)$; prove some continuity properties of its solution-set multifunction, and establish further existence results for mappings which are approximable in some sense. 
Proposition 6.1. Let $d>0, c \in \mathcal{C}, q^{0} \in \mathbb{R}^{n}$, and $\Phi^{0} \in \mathcal{X}$.

If $q^{0} \in\left[\mathcal{S}\left(0, \Phi^{0}\right)\right]^{\#}$ (resp. $\left.\mathrm{V}_{q^{0}}\left(\Phi^{0}\right)=\{0\}\right)$, then there exists $\varepsilon>0$ such that for all $q \in \mathbb{R}^{n}$ and all $\Phi \in \mathcal{X}$ which are c-subhomogeneous (resp. simply cuscos) satisfying

$$
\left\|q-q^{0}\right\|+\mathrm{dI}\left(\Phi, \Phi^{0}\right)<\varepsilon,
$$

one has $q \in[\mathcal{S}(0, \Phi)]^{\#}\left(\right.$ resp. $\left.\mathrm{V}_{q}(\Phi)=\{0\}\right)$.

Proof. We first consider the case $q^{0} \in\left[\mathrm{S}\left(0, \Phi^{0}\right)\right]^{\#}$. Suppose on the contrary, that there exist sequences $\left\{q^{k}, \Phi^{k}, v^{k}\right\}$ satisfying $q^{k} \rightarrow q^{0}, \mathbb{d}\left(\Phi^{k}, \Phi^{0}\right) \rightarrow 0,0 \neq v^{k} \in \mathcal{S}\left(0, \Phi^{k}\right)$, and $\left\langle q^{k}, v^{k}\right\rangle \leq 0$ with $\Phi^{k} \in \mathcal{X}$ being $c$-subhomogeneous. By $c$-subhomogeneity we may assume that $\left\|v^{k}\right\|_{d}=1$, therefore up to subsequences $v^{k} \rightarrow v$ and $\|v\|_{d}=1$. Moreover, for all $k$, there exist $w^{k} \in \Phi^{k}\left(v^{k}\right)$ such that $w^{k} \geq 0$ and $\left\langle v^{k}, w^{k}\right\rangle=0$. By the uniformity in graphical convergence $\Phi^{k} \stackrel{g}{\rightarrow} \Phi^{0}$ [23], Theorem 5.34, we may also assume that $w^{k} \rightarrow w$. From (a) of Theorem 5.37 in [23], it follows in particular that $w \in \Phi^{0}(v)$. Furthermore, $w \geq 0$ and $\langle w, v\rangle=0$. Hence $0 \neq v \in \mathcal{S}\left(0, \Phi^{0}\right)$ and $\left\langle q^{0}, v\right\rangle \leq 0$, contradicting the choice of $q^{0}$.

We now consider the case $\mathrm{V}_{q^{0}}\left(\Phi^{0}\right)=\{0\}$. Suppose on the contrary that there exist sequences $\left\{q^{k}, \Phi^{k}, v^{k}\right\}$ satisfying $q^{k} \rightarrow q^{0}, \mathbb{d I}\left(\Phi^{k}, \Phi^{0}\right) \rightarrow 0$, and $0 \neq v^{k} \in \mathrm{V}_{q^{k}}\left(\Phi^{k}\right)$, with $\Phi^{k} \in \mathcal{X}$. We may assume that $\left\|v^{k}\right\|_{d}=1$, therefore up to subsequences $v^{k} \rightarrow v$ and $\|v\|_{d}=1$. Let us fix $x \geq 0$ and $y \in \Phi^{0}(x)$. Since $\Phi^{k}$, $\Phi^{0}$ are closedvalued and $\Phi^{k} \stackrel{g}{\rightarrow} \Phi^{0}$, we invoke Theorem 5.37 in [23] to obtain $x$ as the limit of a sequence $\left\{a^{j}\right\}$, corresponding to some $\left\{b^{j}\right\}$ satisfying $b^{j} \in \Phi^{j}\left(a^{j}\right)$ and $b^{j} \rightarrow y$. By the choice of $v^{j}$, we obtain $\left\langle b^{j}+q^{j}, v^{j}\right\rangle \leq 0$. Thus $\langle y+q, v\rangle \leq 0$, and therefore $0 \neq v \in \mathrm{V}_{q^{0}}\left(\Phi^{0}\right)$ a contradiction.

Theorem 6.2. Let $d>0, c \in \mathcal{C}, q^{0} \in \mathbb{R}^{n}$, and $\Phi^{0} \in \mathcal{X}$.

If $q^{0} \in\left[\mathcal{S}\left(0, \Phi^{0}\right)\right]^{\#}$ (resp. $\left.\quad \mathrm{V}_{q^{0}}\left(\Phi^{0}\right)=\{0\}\right)$, then there exists $\varepsilon>0$ such that for all $q \in \mathbb{R}^{n}$ and all $\Phi \in \mathcal{X}$ which are c-subhomogeneous and either $\mathbf{G T}(d)$ or $\mathbf{G} \tilde{\mathbf{T}}(d)$ (resp. simply q-pseudomonotone) satisfying $\left\|q-q^{0}\right\|+\mathrm{d}\left(\Phi, \Phi^{0}\right)<\varepsilon$, the set $\mathcal{S}(q, \Phi)$ is nonempty and compact.

Proof. This follows from the above proposition and Corollary 5.3 and Theorem 5.8.

The following theorem may be considered as a stability result for $\operatorname{MCP}\left(q^{0}, \Phi^{0}\right)$ under a copositivity and $c$-subhomogeneous or $q$-pseudomonotone perturbation. Notice it is only required that $\Phi^{0} \in \mathcal{X}$. This theorem extends Theorem 7.5.1 of [4], where only the copositive linear case is considered.

Theorem 6.3. Let $d>0, c \in \mathcal{C}, q^{0} \in \mathbb{R}^{n}$ and $\Phi^{0} \in \mathcal{X}$.

If $q^{0} \in\left[\mathcal{S}\left(0, \Phi^{0}\right)\right]^{\#}\left(\right.$ resp. $\left.\mathrm{V}_{q^{0}}\left(\Phi^{0}\right)=\{0\}\right)$, then there exist $\varepsilon>0$ and $r>0$ such that for all $q \in \mathbb{R}^{n}$ and all $\Phi \in \mathcal{X}$ which are copositive c-subhomogeneous (resp. simply q-pseudomonotone) the following implication holds

$$
\left\|q-q^{0}\right\|+\mathrm{d}\left(\Phi, \Phi^{0}\right)<\varepsilon \Longrightarrow\|x\| \leq r \quad \text { for all } x \in \mathcal{S}(q, \Phi) .
$$

Proof. By Theorem 6.2 for such $q$ and $\Phi$ the set $\mathcal{S}(q, \Phi)$ is nonempty and compact.

Suppose on the contrary that there exist sequences $\left\{q^{k}, \Phi^{k}, x^{k}\right\}$ satisfying $q^{k} \rightarrow q^{0}$, dI $\left(\Phi^{k}, \Phi^{0}\right) \rightarrow 0, x^{k} \in$ $\mathcal{S}\left(q^{k}, \Phi^{k}\right)$, and $\left\langle d, x^{k}\right\rangle \rightarrow+\infty$. There exists a sequence $\left\{y^{k}\right\}$ such that for all $k$

$$
y^{k} \in \Phi^{k}\left(x^{k}\right), \quad y^{k}+q^{k} \geq 0, \quad \text { and } \quad\left\langle y^{k}+q^{k}, x^{k}\right\rangle=0 .
$$

Setting $\sigma_{k}=\left\langle d, x^{k}\right\rangle$, up to subsequences, $\frac{x^{k}}{\sigma_{k}} \rightarrow v \neq 0$. Clearly $\left(x^{k}, y^{k}\right)$ is a solution of problem (PMVIP $\left.{ }_{\mathrm{k}}\right)$ for $\Phi=\Phi^{0}, \Psi^{k}=0$, and $q=q^{0}$ for each $k$, so we can apply the basic lemma (for $\Phi=\Phi^{0}, \Psi^{k}=0$, and $q=q^{0}$ for all $k$ ).

If $\Phi^{k}$ is copositive $c$-subhomogeneous for all $k$, by (d) of the basic lemma and (6.1) we obtain that $0 \neq v \in$ $\mathcal{S}\left(0, \Phi^{0}\right)$ and $\left\langle q^{k}, x^{k}\right\rangle \leq 0$, thus $\left\langle q^{0}, v\right\rangle \leq 0$ contradicting the choice of $q^{0}$.

If $\Phi^{k}$ is $q^{k}$-pseudomonotone for all $k$. By (f) of the basic lemma $0 \neq v \in \mathrm{V}_{q^{0}}\left(\Phi^{0}\right)$ a contradiction. 
In what follows, we recall another type of continuity for multifunctions. Let $X, Y$ be two metric spaces. The mapping $\mathcal{F}: X \hookrightarrow Y$ is said to be outer semicontinuous (osc) at $\bar{x}$ if,

$$
\limsup _{x \rightarrow \bar{x}} \mathcal{F}(x) \subseteq \mathcal{F}(\bar{x})
$$

or equivalently $\limsup _{x \rightarrow \bar{x}} \mathcal{F}(x)=\mathcal{F}(\bar{x})$ where

$$
\limsup _{x \rightarrow \bar{x}} \mathcal{F}(x)=\left\{z: \liminf _{x \rightarrow \bar{x}} d_{\mathcal{F}(x)}(z)=0\right\} .
$$

On $\mathbb{R}^{n} \times \mathcal{X}$ we introduce the metric

$$
D\left(\left(q_{1}, \Phi_{1}\right)\left(q_{2}, \Phi_{2}\right)\right) \doteq\left\|q_{1}-q_{2}\right\|+\mathrm{dI}\left(\Phi_{1}, \Phi_{2}\right) .
$$

Obviously $\left(\mathbb{R}^{n} \times \mathcal{X}, D\right)$ is a metric space. We now investigate continuity properties of the solution-set multifunction $\mathcal{S}: \mathbb{R}^{n} \times \mathcal{X} \hookrightarrow \mathbb{R}^{n}$.

Theorem 6.4. Let $\mathcal{S}$ be the solution-set multifunction associated to problem (MCP). Then $\mathcal{S}$ is osc at each $\left(q^{0}, \Phi^{0}\right)$.

Proof. We have to prove

$$
\limsup _{(q, \Phi) \rightarrow\left(q^{0}, \Phi^{0}\right)} \mathcal{S}(q, \Phi) \subseteq \mathcal{S}\left(q^{0}, \Phi^{0}\right)
$$

If the set in the left-hand of (6.2) is empty, the assertion is trivial. On the contrary, let $x$ be in that set, then there exist a sequence $\left(q^{k}, \Phi^{k}\right) \in \mathbb{R}^{n} \times \mathcal{X}$ such that $q^{k} \rightarrow q^{0}$, dI $\left(\Phi^{k}, \Phi^{0}\right) \rightarrow 0$, and a sequence $x^{k} \rightarrow x$ with $x^{k} \in \mathcal{S}\left(q^{k}, \Phi^{k}\right)$. Thus, $x^{k} \geq 0$ and there is $y^{k} \in \Phi^{k}\left(x^{k}\right)$ such that $y^{k}+q^{k} \geq 0$ and $\left\langle y^{k}+q^{k}, x^{k}\right\rangle=0$. By the uniformity in graphical convergence, we conclude that $\left\{y^{k}\right\}$ is bounded, and so, up to subsequences, we may assume $y^{k} \rightarrow y$. From Theorem 5.37 in [23], it follows that $y \in \Phi^{0}(x)$. Taking the limit we obtain $x \geq 0$, $y+q^{0} \geq 0$, and $\left\langle y+q^{0}, x\right\rangle=0$, that is, $x \in \mathcal{S}\left(q^{0}, \Phi^{0}\right)$.

It is worth mentioning that by Theorem 6.2 we may ensure the nonemptiness of the left-hand side of (6.2) by considering that $q^{0} \in\left[\mathcal{S}\left(0, \Phi^{0}\right)\right]^{\#}$ (resp. $\mathrm{V}_{q^{0}}\left(\Phi^{0}\right)=\{0\}$ ) and restricting $\Phi$ to be $c$-subhomogeneous and either $\mathrm{GT}(d)$ or $\mathrm{G} \tilde{\mathrm{T}}(d)$ for some $c \in \mathcal{C}$ and $d>0$ (resp. $q$-pseudomonotone).

We now provide existence results for other classes of multifunctions, which admit some kind of approximating mappings.

Definition 6.5. Let $\mathcal{Y}$ be any class of multifunctions, the mapping $\Phi \in \mathcal{X}$ is said to be approximable by $\mathcal{Y}$ if there exists a sequence $\left\{\Phi^{k}\right\} \subseteq \mathcal{Y} \cap \mathcal{X}$ such that $\Phi^{k} \stackrel{g}{\rightarrow} \Phi$.

Some properties of the approximating sequence $\left\{\Phi^{k}\right\}$ can be inherit by the approximable mapping $\Phi$, as shown in the next result.

Proposition 6.6. Let $d>0, c \in \mathcal{C}$ and $\Phi,\left\{\Phi^{k}\right\}$ in $\mathcal{X}$ such that $\Phi^{k} \stackrel{g}{\rightarrow} \Phi$ :

(a) if $0 \in \Phi^{k}(0)$ for each $k$ then $0 \in \Phi(0)$;

(b) if $\left\{\Phi^{k}\right\}$ are monotone (resp. copositive) then $\Phi$ is monotone (resp. copositive);

(c) if $\left\{\Phi^{k}\right\}$ are c-subhomogeneous (resp. c-Moré) then $\Phi$ is c-subhomogeneous (resp. c-Moré) provided c is continuous;

(d) if $\left\{\Phi^{k}\right\}$ are zero-subhomogeneous then $\Phi$ is zero-subhomogeneous;

Proof.

(a) It follows from Theorem 5.37 in [23].

(b) For the monotone case see [23], Theorem 12.32. For the copositive case is similar. 
(c) Let $x \in \Delta_{d}, \lambda>0$ (resp. $\lambda \geq 1$ ) and $y \in \Phi(\lambda x)$. By Theorem 5.37(b) in [23] there exist sequences $\left\{u^{k}\right\} \subseteq \mathbb{R}_{+}^{n}$ and $\left\{y^{k}\right\}$ such that $u^{k} \rightarrow \lambda x, y^{k} \in \Phi^{k}\left(u^{k}\right)$, and $y^{k} \rightarrow y$ (we may assume that $u^{k} \neq 0$ ). Clearly, $\frac{y^{k}}{c\left(\left\|u^{k}\right\|_{d}\right)} \rightarrow \frac{y}{c(\lambda)}$ and $\frac{u^{k}}{\left\|u^{k}\right\|_{d}} \rightarrow x$.

If $\left\{\Phi^{k}\right\}$ are $c$-subhomogeneous, $\Phi^{k}\left(u^{k}\right) \subseteq c\left(\left\|u^{k}\right\|_{d}\right) \Phi^{k}\left(\frac{u^{k}}{\left\|u^{k}\right\|_{d}}\right)$, thus $\frac{y^{k}}{c\left(\left\|u^{k}\right\|_{d}\right)} \in \Phi^{k}\left(\frac{u^{k}}{\left\|u^{k}\right\|_{d}}\right)$. By taking the limit and applying Theorem 5.37(a) in [23] we obtain $\frac{y}{c(\lambda)} \in \Phi(x)$, thus $y \in c(\lambda) \Phi(x)$.

If $\left\{\Phi^{k}\right\}$ are $c$-Moré, there exists $z^{k} \in \Phi^{k}\left(\frac{u^{k}}{\left\|u^{k}\right\|_{d}}\right)$ such that $\left\langle y^{k}, u^{k}\right\rangle \geq c\left(\left\|u^{k}\right\|_{d}\right)\left\langle z^{k}, u^{k}\right\rangle$. By the uniformity in graphical convergence [23], Theorem 5.34, and Theorem 5.37 in [23], there exists $z$ such that (up to subsequences) $z^{k} \rightarrow z \in \Phi(x)$, and by taking the limit in the previous inequality we get $\langle y, \lambda x\rangle \geq c(\lambda)\langle z, x\rangle$.

(d) It is similar as in (c).

Example 6.7. It is worth pointing out that if $\left\{\Phi^{k}\right\}$ are $q$-pseudomonotone then $\Phi$ is not necessarily $q$ pseudomonotone. To see this take $\Phi^{k}(x)=M x+b^{k}, \Phi(x)=M x$ and $q=0$ where

$$
M=\left(\begin{array}{cc}
0 & 1 \\
0 & 0
\end{array}\right) \quad \text { and } \quad b^{k}=\left(\begin{array}{c}
\frac{1}{k} \\
0
\end{array}\right),
$$

each $\Phi^{k}$ is 0-pseudomonotone, $\Phi^{k} \stackrel{g}{\rightarrow} \Phi$ (see [23], p. 353), and $\Phi$ is not 0-pseudomonotone since $\left\langle M e^{1}, e^{2}-e^{1}\right\rangle=$ 0 and $\left\langle M e^{2}, e^{2}-e^{1}\right\rangle<0$.

Theorem 6.8. Let $d>0, c \in \mathcal{C}$, and $q \in \mathbb{R}^{n}$.

(a) Let $\Phi=\Phi^{0}+\Psi^{0}$ where $\Phi^{0}, \Psi^{0} \in \mathcal{X}$ the latter being copositive and zero-subhomogeneous:

(a.1) If $\Phi^{0}$ is approximable by copositive c-subhomogeneous mappings and $q \in\left[\mathcal{S}\left(0, \Phi^{0}\right)\right]^{\#}$ then $\mathcal{S}(q, \Phi)$ is nonempty;

(a.2) If $\Phi^{0}$ is approximable by copositive c-Moré mappings and $q \in\left[\mathrm{U}_{0}\left(\Phi^{0}\right)\right]^{\#}$ then $\mathcal{S}(q, \Phi)$ is nonempty;

(b) If $\Phi \in \mathcal{X}$ is approximable by q-pseudomonotone mappings and $\mathrm{V}_{q}(\Phi)=\{0\}$ then $\mathcal{S}(q$, $\Phi)$ is nonempty.

Proof.

(a) Let $\left\{\Phi^{k}\right\}$ be the mappings that approximate $\Phi^{0},\left\{\sigma_{k}\right\}$ be an increasing sequence of positive numbers converging to $+\infty$, and $\left\{\left(x^{k}, y^{k}, r^{k}\right)\right\}$ be a sequence of solutions to $\left(\operatorname{PMVIP}_{\mathrm{k}}\right)$ for $\Phi=\Phi^{0}, \Psi^{k}=\Psi=\Psi^{0}$, and $q^{k}=q$ for all $k$.

If $\left\{x^{k}\right\}$ is bounded, by the uniformity in graphical convergence [23], Theorem 5.34, and Theorem 5.37 in [23], any limit point of such a sequence belongs to $\mathcal{S}(q, \Phi)$.

Otherwise, we may consider (by redefining $\sigma_{k}$ if necessary) that $\sigma_{k}=\left\langle d, x^{k}\right\rangle$. Since $\left\langle d, \frac{x^{k}}{\sigma_{k}}\right\rangle=1$, up to subsequences, there exists $0 \neq v \geq 0$ such that $\frac{x^{k}}{\sigma_{k}} \rightarrow v$. By applying the basic lemma (for $\Phi=\Phi^{0}$, $\Psi^{k}=\Psi=\Psi^{0}$, and $q^{k}=q$ for all $\left.k\right)$ we obtain that:

(a.1) there exist $w \in \Phi^{0}(v), r \in \Psi^{0}(v)$, and $\emptyset \neq J_{v} \subset I$, such that $0 \neq v \in \mathcal{S}\left(-\langle w, v\rangle d, \Phi^{0}\right)$ and $\langle w, v\rangle \leq 0$. As $\Phi^{0}$ is copositive (Proposition 6.6(b)) we conclude that $\langle w, v\rangle=0$ and $0 \neq v \in \mathcal{S}\left(0, \Phi^{0}\right)$. By the copositivity of $\Phi^{k}$ and $\Psi^{0}$ we obtain $\left\langle y^{k_{m}}, x^{k_{m}}\right\rangle \geq 0$ and $\left\langle r^{k_{m}}, x^{k_{m}}\right\rangle \geq 0$ respectively. By setting $x=0$ in $\left(\mathrm{PMVIP}_{\mathrm{k}}\right)$ we get

$$
\left\langle q, \frac{x^{k_{m}}}{\sigma_{k_{m}}}\right\rangle \leq\left\langle y^{k_{m}}+r^{k_{m}}+q, \frac{x^{k_{m}}}{\sigma_{k_{m}}}\right\rangle \leq 0,
$$

then $\langle q, v\rangle \leq 0$ contradicting the choice of $q$;

(a.2) there exist $w$ and $w^{k_{m}} \in \Phi^{k_{m}}\left(\frac{x^{k_{m}}}{\sigma_{k_{m}}}\right)$ such that $w^{k_{m}} \rightarrow w \in \Phi^{0}(v)$ and $\langle w, v\rangle \leq 0$. Since $\Phi^{0}$ is copositive (Prop. 6.6(b)) we get $\langle w, v\rangle=0$, thus $0 \neq v \in \mathrm{U}_{0}\left(\Phi^{0}\right)$. As above we get $(6.3)$ and thus $\langle q, v\rangle \leq 0$ contradicting the choice of $q$.

(b) The result follows from Theorem 6.3 and the uniformity in graphical convergence [23], Theorems 5.34 and $5.37(\mathrm{a})$. 


\section{Remark 6.9.}

1. If we set $\Psi^{0}=0$ in $(a .1)$, then the result follows from Theorem 6.3 (similarly as in the proof of $(b)$ ).

2. In Theorem $6.8(b)$ the solution set may be unbounded as shown by Example 6.7 where $\mathcal{S}(q, \Phi)=\left\{\left(x_{1}, 0\right)^{\mathrm{T}}\right.$ : $\left.x_{1} \geq 0\right\}$. For this example we can use neither Theorem 5.8 nor Theorem 5.9, but by using $(b)$ we get the existence of solutions. 3. If in $(a)$ we additionally assume that $c$ is continuous, then by Proposition $6.6(b)-(c)$ and Theorem 5.2 or 5.5 respectively, the set $\mathcal{S}(q, \Phi)$ is also compact. Similarly, if in $(b)$ we assume that $\Phi$ is approximable by monotone mappings, instead of $q$-pseudomonotone, by Proposition 6.6(b) and Theorem 5.8 the set $\mathcal{S}(q, \Phi)$ is also compact.

4. Notice that under the assumptions of Theorem 6.8 we actually prove that any limit point of every approximate sequence $\left\{x^{k}\right\}$ constructed through $\left(\mathrm{PMVIP}_{\mathrm{k}}\right)$ (which is bounded) is a solution to (MCP).

\section{Estimates for the SOlution SET}

In this section we extend and generalize some results from [17], where the monotone linear complementarity problem is studied; [14], where the numerical range of an operator is used to obtain bounds for the linear complementarity problem in Hilbert spaces; and [21], where a bound for the solution set of a quasidifferentiable convex programming problem is obtained. Indeed, we consider the set-valued case and obtain bounds for the solution set to problem (MCP) mainly under the subhomogeneity property.

If $\Phi \in \mathcal{X}$ is $c$-subhomogeneous (on $\Delta_{d}$ ), we infer that

$$
m_{\Phi}\|x\|_{d} c\left(\|x\|_{d}\right) \leq\langle y, x\rangle \leq M_{\Phi}\|x\|_{d} c\left(\|x\|_{d}\right) \quad \text { for all } x \geq 0, y \in \Phi(x) .
$$

In the following $\|\cdot\|$ and $\|\cdot\|_{1}$ denote the Euclidean norm and the sum-norm in $\mathbb{R}^{n}$ respectively, and $d_{0} \doteq$ $\min _{1 \leq i \leq n} d_{i}>0$. We point out that $\Psi$ is uniformly bounded if and only if $\|\Psi\|<+\infty$, where $\|\Psi\| \doteq$ $\sup \{\|y\|: y \in \Psi(x), x \geq 0\}$.

Theorem 7.1. Let $d>0, c \in \mathcal{C}, q \in \mathbb{R}^{n}$, and $\Phi, \Psi \in \mathcal{X}$.

(a) Assume $\Phi$ is c-subhomogeneous and $\Psi$ is uniformly bounded:

- if $M_{\Phi}<0$, then $\mathcal{S}(q, \Phi+\Psi) \subseteq\left\{x \geq 0: c\left(\|x\|_{d}\right) \leq \max \left(c(0), \frac{\|\Psi\|+\|q\|}{d_{0}\left|M_{\Phi}\right|}\right)\right\}$;

- if $m_{\Phi}>0$, then $\mathcal{S}(q, \Phi+\Psi) \subseteq\left\{x \geq 0: c\left(\|x\|_{d}\right) \leq \max \left(c(0), \frac{\|\Psi\|+\|q\|}{d_{0} m_{\Phi}}\right)\right\}$.

(b) Assume $\Phi$ is monotone, $\Psi$ is copositive zero-subhomogeneous, and there exist $0 \neq x^{0} \geq 0, y^{0} \in \Phi\left(x^{0}\right)$ such that $y^{0}+q>0$, then

$$
\mathcal{S}(q, \Phi+\Psi) \subseteq\left\{x \geq 0:\|x\|_{1} \leq \frac{\left\langle y^{0}+q, x^{0}\right\rangle+\|\Psi\|\left\|x^{0}\right\|}{\min _{1 \leq i \leq n}\left(y^{0}+q\right)_{i}}\right\} .
$$

Proof.

(a) First we notice that for $x \geq 0$ and $r \in \Psi(x),\|r\| \leq\|\Psi\|$. Assume $M_{\Phi}<0$, and let $0 \neq x \geq 0, y \in \Phi(x)$, and $r \in \Psi(x)$. By $(7.1)$

$$
\langle y+r+q, x\rangle \leq M_{\Phi}\|x\|_{d} c\left(\|x\|_{d}\right)+(\|r\|+\|q\|)\|x\| \leq\|x\|_{d}\left(M_{\Phi} c\left(\|x\|_{d}\right)+\frac{1}{d_{0}}(\|\Psi\|+\|q\|)\right) .
$$

It follows that $M_{\Phi} c\left(\|x\|_{d}\right)+\frac{1}{d_{0}}(\|\Psi\|+\|q\|) \geq 0$ if $0 \neq x \in \mathcal{S}(q, \Phi+\Psi)$.

Let $m_{\Phi}>0,0 \neq x \geq 0, y \in \Phi(x)$, and $r \in \Psi(x)$. By (7.1) again

$$
\langle y+r+q, x\rangle \geq m_{\Phi}\|x\|_{d} c\left(\|x\|_{d}\right)-(\|r\|+\|q\|)\|x\| \geq\|x\|_{d}\left(m_{\Phi} c\left(\|x\|_{d}\right)-\frac{1}{d_{0}}(\|\Psi\|+\|q\|)\right) .
$$

It follows that $m_{\Phi} c\left(\|x\|_{d}\right)-\frac{1}{d_{0}}(\|\Psi\|+\|q\|) \leq 0$ if $0 \neq x \in \mathcal{S}(q, \Phi+\Psi)$. 
(b) Let $x \in \mathcal{S}(q, \Phi+\Psi)$, there exist $y \in \Phi(x)$ and $r \in \Psi(x)$ such that $\langle y+r+q, u-x\rangle \geq 0$ for all $u \geq 0$, since $\Phi$ is $(r+q)$-pseudomonotone we get $\left\langle y^{0}+r+q, x^{0}-x\right\rangle \geq 0$ and since $\Psi$ is copositive

$$
\left\langle y^{0}+r+q, x^{0}\right\rangle \geq\left\langle y^{0}+r+q, x\right\rangle \geq\left\langle y^{0}+q, x\right\rangle \geq \min _{1 \leq i \leq n}\left(y^{0}+q\right)_{i}\|x\|_{1} .
$$

By Proposition 2.3(b) $\Psi$ is uniformly bounded and $\left\langle r, x^{0}\right\rangle \leq\|\Psi\|\left\|x^{0}\right\|$. Thus

$$
\left\langle y^{0}+q, x^{0}\right\rangle+\|\Psi\|\left\|x^{0}\right\| \geq \min _{1 \leq i \leq n}\left(y^{0}+q\right)_{i}\|x\|_{1}
$$

Remark 7.2. One can check that hypothesis $m_{\Phi}>0$ in $(a)$ implies that $\Phi \in \mathbf{G} \tilde{\mathbf{T}}(d)$ and $\mathcal{S}(0, \Phi)=\{0\}$, which in turn implies that $\mathcal{S}(q, \Phi+\Psi)$ is nonempty and compact for all $q \in \mathbb{R}^{n}$ provided $\Psi$ is copositive (Th. 5.2). Similarly, the hypothesis in (b) implies that $\mathcal{S}(q, \Phi+\Psi)$ is nonempty and compact provided $\Phi$ is copositive as well (Th. 5.8 implication $(a) \Rightarrow(b)$ and Th. 5.7).

Corollary 7.3. Let $d>0, c \in \mathcal{C}, q \in \mathbb{R}^{n}$, and $\Phi \in \mathcal{X}$.

(a) Assume $\Phi$ is c-subhomogeneous:

- if $M_{\Phi}<0$, then $\mathcal{S}(q, \Phi) \subseteq\left\{x \geq 0: c\left(\|x\|_{d}\right) \leq \max \left(c(0), \frac{\|q\|}{d_{0} \mid M_{\Phi}}\right)\right\}$;

- if $m_{\Phi}>0$, then $\mathcal{S}(q, \Phi) \subseteq\left\{x \geq 0: c\left(\|x\|_{d}\right) \leq \max \left(c(0), \frac{\|q\|}{d_{0} m_{\Phi}}\right)\right\}$.

(b) Assume $\Phi$ is q-pseudomonotone, and there exist $0 \neq x^{0} \geq 0, y^{0} \in \Phi\left(x^{0}\right)$ such that $y^{0}+q>0$, then

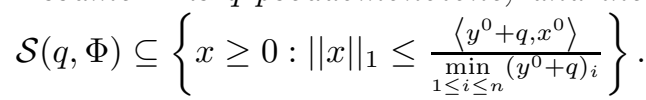

Proof.

(a) We set $\Psi=0$ in (a) of the above theorem.

(b) We proceed as in (b) of the above theorem with $\Psi=0$, and taking into account that $\Phi$ is $q$-pseudomonotone.

Remark 7.4. We point out that $m_{\Phi}>0$ in $(a)$ implies that $\Phi \in \mathbf{G} \tilde{\mathbf{T}}(d)$ and $\mathcal{S}(0, \Phi)=\{0\}$, which in turn implies that $\mathcal{S}(q, \Phi)$ is nonempty and compact for all $q \in \mathbb{R}^{n}$ (Cor. 5.3). Similarly, the hypothesis in (b) implies that $\mathcal{S}(q, \Phi)$ is nonempty and compact (Th. 5.8).

Acknowledgements. The authors are grateful to both referees for their useful remarks on a first draft of the paper.

\section{REFERENCES}

[1] J.-P. Aubin and A. Cellina, Differential Inclusions. Springer, Berlin (1984).

[2] J.-P. Aubin and H. Frankowska, Set-Valued Analysis. Birkhäuser, Boston (1990).

[3] A. Auslender and M. Teboulle, Asymptotic Cones and Functions in Optimization and Variational Inequalities. Springer, Berlin (2003).

[4] R.W. Cottle, J.S. Pang and R.E. Stone, The Linear Complementarity Problem. Academic Press, New York (1992).

[5] J.P. Crouzeix, Pseudomonotone variational inequality problems: Existence of solutions. Math. Program. 78 (1997) 305-314.

[6] A. Daniilidis and N. Hadjisavvas, Coercivity conditions and variational inequalities. Math. Program. 86 (1999) $433-438$.

[7] F. Flores-Bazán, Existence theorems for generalized noncoercive equilibrium problems: the quasi-convex case. SIAM J. Optim. 11 (2000) 675-690.

[8] F. Flores-Bazán, Existence theory for finite dimensional pseudomonotone equilibrium problems. Acta Appl. Math. 77 (2003) 249-297.

[9] F. Flores-Bazán and R. López, The linear complementarity problem under asymptotic analysis. Math. Oper. Res. 30 (2005) 73-90.

[10] C.B. García, Some classes of matrices in linear complementarity theory. Math. Program. 5 (1973) 299-310.

[11] S.M. Gowda, Complementarity problems over locally compact cones. SIAM J. Control Optim. 27 (1989) 836-841.

[12] S.M. Gowda and J.-S. Pang, The basic theorem of complementarity revisited. Math. Program. 58 (1993) 161-177. 
[13] S.M. Gowda and J.-S. Pang, Some existence results for multivalued complementarity problems. Math. Oper. Res. 17 (1992) 657-669.

[14] G. Isac, The numerical range theory and boundedness of solutions of the complementarity problem. J. Math. Anal. Appl. 143 (1989) 235-251.

[15] S. Karamardian, The complementarity problem. Math. Program. 2 (1972) 107-129.

[16] S. Karamardian, An existence theorem for the complementarity problem. J. Optim. Theory Appl. 19 (1976) $227-232$.

[17] O.L. Mangasarian and L. McLinden, Simple bounds for solutions of monotone complementarity problems and convex programs. Math. Program. 32 (1985) 32-40.

[18] J.J. Moré, Classes of functions and feasibility conditions in nonlinear complementarity problems. Math. Program. 6 (1974) $327-338$.

[19] J.J. Moré, Coercivity conditions in nonlinear complementarity problems. SIAM Rev. 17 (1974) 1-16.

[20] J. Parida and A. Sen, Duality and existence theory for nondifferenciable programming. J. Optim. Theory Appl. 48 (1986) 451-458.

[21] J. Parida and A. Sen, A class of nonlinear complementarity problems for multifunctions. J. Optim. Theory Appl. 53 (1987) $105-113$.

[22] J. Parida and A. Sen, A variational-like inequality for multifunctions with applications. J. Math. Anal. Appl. 124 (1987) 73-81.

[23] R.T. Rockafellar and R.J.-B. Wets, Variational Analysis. Springer, Berlin (1998).

[24] R. Saigal, Extension of the generalized complementarity problem. Math. Oper. Res. 1 (1976) 260-266.

[25] Y. Zhao, Existence of a solution to nonlinear variational inequality under generalized positive homogeneity. Oper. Res. Lett. 25 (1999) 231-239. 